\title{
Supporting Information \\ Enol Esters: Versatile Substrates for \\ Mannich-type Multicomponent Reactions
}

\begin{abstract}
Nicolas Isambert, Montse Cruz, María José Arévalo, Elena Gómez and Rodolfo Lavilla
Institute for Research in Biomedicine, Barcelona Science Park, Josep Samitier 1-5, 08028 Barcelona (SPAIN) and Laboratory of Organic Chemistry, Faculty of Pharmacy, University of Barcelona (SPAIN)
\end{abstract}

\section{General Procedure}

Enol ester 1 ( $1 \mathrm{mmol}, 1$ eq.) was added to a suspension containing aniline 2 ( $1 \mathrm{mmol}$, 1eq.), aldehyde 3 ( $1 \mathrm{mmol}, 1$ eq.), scandium triflate ( $0.2 \mathrm{mmol}, 0.2$ eq.) and activated molecular sieves $(2 \mathrm{~g}, 4 \AA)$ in acetonitrile $(5 \mathrm{ml})$, and the resulting mixture was stirred at room temperature overnight under inert atmosphere. To the resulting solution was added pyridine ( $3 \mathrm{mmol}, 3$ eq.) and thionyl chloride (1.2 mmol, 1,2 eq.) at $0{ }^{\circ} \mathrm{C}$, this temperature was kept for 1 hour and the reaction was allowed to warm up to room temperature and stirred for 3 hours. Then, trifluoroacetic acid $(0.2 \mathrm{mmol}, 0.2$ eq. $)$ was added to the reaction mixture and the solution was heated to reflux for 1 day. The reaction mixture was allowed to cool down, quenched with a solution of $\mathrm{HCl}(1 \mathrm{~N})(20$ $\mathrm{mL})$ and extracted with ethyl acetate $(3 \times 15 \mathrm{~mL})$. The combined organic phases were dried over magnesium sulphate, filtered and the solvent was removed in vacuo. The oily residue was purified by flash chromatography eluting with mixture of ethyl acetate/hexane to afford the desired products. Under these conditions, a 1:9 cis/trans ratio is usually obtained. Additional purification is needed for having analytical samples, and this may be achieved by preparative HPLC or flash column chromatography.

- When the TFA treatment is omitted, the lactams 4 are obtained as a 3:1 cis/trans mixtures in roughly the same yield.

- If 3 equivalents of the enol ester $\mathbf{1}$ are added, the overall yield is slightly increased (4a, $45 \%)$

- When $\mathrm{Yb}(\mathrm{OTf})_{3}$ is used as the catalyst, and TMSCl (1,1 eq) is added, the overall yield increases (4d, 65\%). 


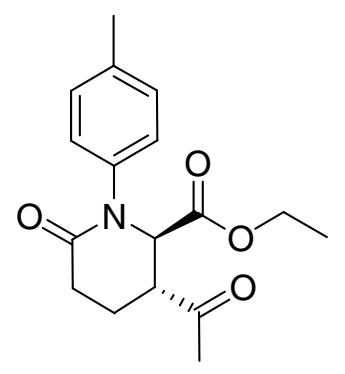

Lactam 4a was obtained as an oil $(40 \%) .{ }^{1} \mathrm{H}$ NMR $\left(400 \mathrm{MHz}, \mathrm{CDCl}_{3}\right) \delta{ }^{1} \mathrm{H}$ NMR : 7.25 $(\mathrm{d}, J=7.3 \mathrm{~Hz}, 2 \mathrm{H}), 7.18(\mathrm{~d}, J=7.1 \mathrm{~Hz}, 2 \mathrm{H}), 4.90(\mathrm{dd}, J=2.5,0.8 \mathrm{~Hz}, 1 \mathrm{H}), 4.17(\mathrm{~m}$, $2 \mathrm{H}), 3.22(\mathrm{~m}, J=5.4,2.5 \mathrm{~Hz}, 1 \mathrm{H}), 2.61(\mathrm{~m}, J=17.5,5.8 \mathrm{~Hz}, 1 \mathrm{H}), 2.44(\mathrm{~m}, J=17.5$, 9.2, $6.7 \mathrm{~Hz}, 1 \mathrm{H}), 2.34(\mathrm{~s}, 3 \mathrm{H}), 2.32(\mathrm{~s}, 3 \mathrm{H}), 2.26(\mathrm{~m}, 2 \mathrm{H}), 1.23(\mathrm{t}, J=7.1 \mathrm{~Hz}, 3 \mathrm{H}) ;{ }^{13} \mathrm{C}-$ NMR (100 MHz, $\left.\mathrm{CDCl}_{3}\right) \delta$ 205.2, 171.2, 169.2, 139.4, 137.3, 129.8, 127.3, 62.7, 62.0, 49.1, 29.6, 27.3, 21.1, 21.0, 14.0. IR $\left(\mathrm{KBr}, \mathrm{cm}^{-1}\right)$ 2916, 1734, 1707, 1662, 1483, 1179. MS (EI) $\mathrm{m} / z(\%)$ : $303(\mathrm{M}, 23), 230(33), 188$ (100). HRMS: calcd for $\mathrm{C}_{17} \mathrm{H}_{21} \mathrm{NO}_{4}$, 303.1471; found, 303.1503.

trans-Ethyl 3-acetyl-5-oxo- $N$-p-tolylpyrrolidine-2-carboxylate (trans-4b)

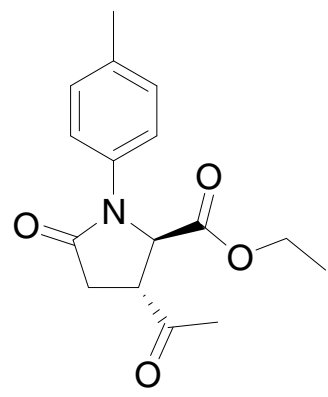

Lactam 4b was obtained as a waxy solid (31\%). ${ }^{1} \mathrm{H}$ NMR $\left(400 \mathrm{MHz}, \mathrm{CDCl}_{3}\right) \delta 7.29(\mathrm{~d}$, $J=8.4 \mathrm{~Hz}, 2 \mathrm{H}), 7.18(\mathrm{~d}, J=8.4 \mathrm{~Hz}, 2 \mathrm{H}), 5.04(\mathrm{~d}, J=3.1 \mathrm{~Hz}, 1 \mathrm{H}), 4.18(\mathrm{q}, J=7.1 \mathrm{~Hz}$, 2H), $3.37(\mathrm{dt}, J=10.2,3.6 \mathrm{~Hz}, 1 \mathrm{H}), 3.09(\mathrm{dd}, J=17.4,10.4 \mathrm{~Hz}, 1 \mathrm{H}), 2.81(\mathrm{dd}, J=$ 17.4, 4.0 Hz, 1H), $2.33(\mathrm{~s}, 6 \mathrm{H}), 1.20(\mathrm{t}, J=7.1 \mathrm{~Hz}, 3 \mathrm{H}) .{ }^{13} \mathrm{C} \mathrm{NMR}\left(100 \mathrm{MHz}, \mathrm{CDCl}_{3}\right)$ $\delta$ 204.4, 172.1, 171.0, 136.8, 134.7, 129.8, 123.6, 62.9, 62.3, 47.6, 33.3, 28.1, 21.1, 14.1. IR $\left(\mathrm{KBr}, \mathrm{cm}^{-1}\right)$ 2934, 1698, 1619, 1510, 1376, 1188. MS (CI) $\mathrm{m} / \mathrm{z}(\%): 290$ $(\mathrm{M}+\mathrm{H})$. HRMS: calcd for $\mathrm{C}_{16} \mathrm{H}_{19} \mathrm{NO}_{4}+\mathrm{H}, 290.1392$; found 290.1406. 
<smiles>CCOC(=O)[C@H]1[C@@H](C(C)=O)CCC(=O)N1c1ccc(OC)cc1</smiles>

Obtained as a white solid in $35 \%$ yield. ${ }^{1} \mathrm{H}-\mathrm{NMR}\left(400 \mathrm{MHz}, \mathrm{CDCl}_{3}\right) \delta 7.15$ (d, $J=8,8$ $\mathrm{Hz}, 2 \mathrm{H}), 6.90(\mathrm{~d}, J=8.8 \mathrm{~Hz}, 2 \mathrm{H}), 4.73(\mathrm{~m}, J=4.8$ and $1.6 \mathrm{~Hz}, 1 \mathrm{H}), 4.13(\mathrm{~m}, 2 \mathrm{H}), 3.80$ (s, 3H), $3.23(\mathrm{~m}, J=12.8,4.4 \mathrm{~Hz}, 1 \mathrm{H}), 2.80(\mathrm{~m}, J=18.8,7.2$ and $2.0 \mathrm{~Hz}, 1 \mathrm{H}), 2.58$ (ddd, $J=18.8,10.8$ and $8.0 \mathrm{~Hz}, 1 \mathrm{H}), 2.33(\mathrm{~s}, 3 \mathrm{H}), 2.17(\mathrm{~m}, 1 \mathrm{H}), 2.07(\mathrm{~m}, 1 \mathrm{H}), 1.19(\mathrm{t}, J$ $=7.2 \mathrm{~Hz}, 3 \mathrm{H}) .{ }^{13} \mathrm{C}-\mathrm{NMR}\left(100 \mathrm{MHz}, \mathrm{CDCl}_{3}\right) \delta 204.6,170.9,169.4,159.2,134.8,128.6$, 114.9, 64.5, 62.4, 55.7, 50.6, 30.7, 28.9, 19.4, 14.1. IR (KBr, $\left.\mathrm{cm}^{-1}\right)$ 2952, 1752, 1707, $1653,1492,1188$. See X-ray diffraction data in the corresponding file.

trans-Ethyl 3-acetyl- $N$-(4-methoxyphenyl)-6-oxopiperidine-2-carboxylate (trans-4c)<smiles>CCOC(=O)[C@H]1[C@@H](C(C)=O)CCC(=O)N1c1ccc(OC)cc1</smiles>

Lactam $4 \mathbf{c}$ was obtained as a sticky solid in $35 \%$ yield. ${ }^{1} \mathrm{H}-\mathrm{NMR}\left(400 \mathrm{MHz}, \mathrm{CDCl}_{3}\right) \delta$ $7.30(J=9.0 \mathrm{~Hz}, 2 \mathrm{H}), 6.90(J=9.0 \mathrm{~Hz}, 2 \mathrm{H}), 4.88(\mathrm{~d}, J=2.4,0.5 \mathrm{~Hz}, 1 \mathrm{H}), 4.15(\mathrm{~m}$, $2 \mathrm{H}), 3.80(\mathrm{~s}, 3 \mathrm{H}), 3.22(\mathrm{dt}, J=8.4,2.4 \mathrm{~Hz}, 1 \mathrm{H}), 2.60(\mathrm{dt}, 17.6,5.6 \mathrm{~Hz}, 1 \mathrm{H}), 2.43$ (ddd, $J$ $=17.6,10.2,5.6 \mathrm{~Hz}, 1 \mathrm{H}), 2.32(\mathrm{~s}, 3 \mathrm{H}), 2.18(\mathrm{~m}, 2 \mathrm{H}), 1.23(\mathrm{t}, J=7.2 \mathrm{~Hz}, 3 \mathrm{H}) .{ }^{13} \mathrm{C} \mathrm{NMR}$ $\left(100 \mathrm{MHz}, \mathrm{CDCl}_{3}\right) \delta 205.5,171.6,169.8,158.9,134.9,128.9,114.7,63.2,62.3,55.6$, 49.3, 28.8, 27.5, 21.3, 14.3. IR (KBr, $\left.\mathrm{cm}^{-1}\right)$ 2925, 1770, 1707, 1608, 1519, 1197. MS (EI) $m / z(\%): 319$ (M, 41), 246 (26), 204 (100); HRMS: calcd for $\mathrm{C}_{17} \mathrm{H}_{21} \mathrm{NO}_{5}, 319,1420$; found $319,1441$. 
trans-Ethyl 3-acetyl- $N$-(4-methoxyphenyl)-5-oxopyrrolidine-2-carboxylate (trans4d)

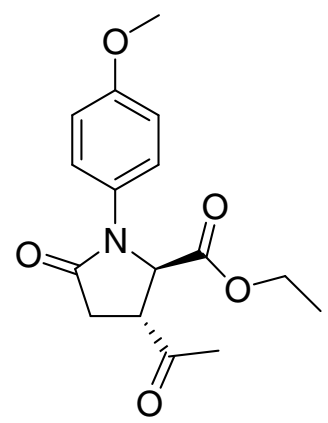

Lactam 4d was obtained as a sticky solid (28\%). ${ }^{1} \mathrm{H}$ NMR $\left(400 \mathrm{MHz}, \mathrm{CDCl}_{3}\right) \delta 7.32(\mathrm{~d}$, $J=9.0 \mathrm{~Hz}, 2 \mathrm{H}), 6.90(\mathrm{~d}, J=9.0 \mathrm{~Hz}, 2 \mathrm{H}), 4.97(\mathrm{~d}, J=3.1 \mathrm{~Hz}, 1 \mathrm{H}), 4.17(\mathrm{~m}, J=7.1$ $\mathrm{Hz}, 2 \mathrm{H}), 3.79$ (s, 3H), $3.35(\mathrm{~m}, J=10.4,4.0,3.1 \mathrm{~Hz}, 1 \mathrm{H}), 3.01(\mathrm{dd}, J=17.2,10.4 \mathrm{~Hz}$, $1 \mathrm{H}), 2.73(\mathrm{dd}, J=17.2,4.0 \mathrm{~Hz}, 1 \mathrm{H}), 2.32(\mathrm{~s}, 3 \mathrm{H}), 1.20(\mathrm{t}, J=7.13 \mathrm{~Hz}, 3 \mathrm{H}) .{ }^{13} \mathrm{C} \mathrm{NMR}$ $\left(100 \mathrm{MHz}, \mathrm{CDCl}_{3}\right) \delta 204.4,171.0,136.8,134.7,129.8,123.6,62.9,62.3,47.6,33.3$, 29.8, 28.1, 21.1, 14.1. IR (KBr, cm $\left.{ }^{-1}\right)$ 2960, 1725, 1615, 1510, 1304, 1122. MS (EI) $\mathrm{m} / \mathrm{z}$ (\%): 305 (M, 41), 232 (25), 190 (100). HRMS: calcd for $\mathrm{C}_{16} \mathrm{H}_{19} \mathrm{NO}_{5}, 305.1263$; found 305. 1286.

trans-Ethyl 3-acetyl- $N$-(4-bromophenyl)-6-oxopiperidine-2-carboxylate (trans-4e)<smiles>CCOC(=O)[C@H]1[C@@H](C(C)=O)CCC(=O)N1c1ccc(Br)cc1</smiles>

Lactam 4e was obtained as a sticky solid (26\%). ${ }^{1} \mathrm{H}$ NMR $\left(400 \mathrm{MHz}, \mathrm{CDCl}_{3}\right) \delta 7.51$ (d, $J=8.6 \mathrm{~Hz}, 2 \mathrm{H}), 7.29(\mathrm{~d}, J=8.6 \mathrm{~Hz}, 2 \mathrm{H}), 4.88(\mathrm{~d}, J=1.58 \mathrm{~Hz}, 1 \mathrm{H}), 4.17(\mathrm{~m}, 2 \mathrm{H}), 3.25$ $(\mathrm{dt}, J=5.4,2.1 \mathrm{~Hz}, 1 \mathrm{H}), 2.59(\mathrm{td}, J=17.2,5.5 \mathrm{~Hz}, 1 \mathrm{H}), 2.40(\mathrm{~m}, 1 \mathrm{H}), 2.32(\mathrm{~s}, 3 \mathrm{H})$, $2.24(\mathrm{~m}, 2 \mathrm{H}), 1.23(\mathrm{t}, J=7.1 \mathrm{~Hz}, 3 \mathrm{H}) .{ }^{13} \mathrm{C} \mathrm{NMR}\left(100 \mathrm{MHz}, \mathrm{CDCl}_{3}\right) \delta 205.9,172.5$, 170.3, 139.7, 132.7, 129.1, 122.6, 62.9, 62.8, 48.4, 28.3, 27.1, 20.0, 13.9; IR (KBr, cm ${ }^{-}$ 1) 2961, 1743, 1707, 1662, 1483, 1188; MS (EI) $\mathrm{m} / \mathrm{z}(\%): 369$ (M, 21), 367 (M, 21), 296 (42), 294 (42), 254 (96), 252 (100); HRMS: calcd for $\mathrm{C}_{16} \mathrm{H}_{18} \mathrm{BrNO}_{4}, 367.0419$; found 367.0401 . 


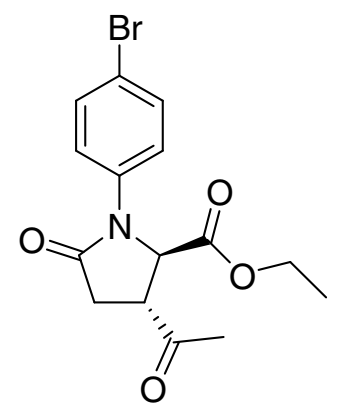

Lactam $4 \mathbf{f}$ was obtained as a sticky solid (25\%). ${ }^{1} \mathrm{H}$ NMR $\left(400 \mathrm{MHz}, \mathrm{CDCl}_{3}\right) \delta 7.55$ $(\mathrm{d}, J=8.7 \mathrm{~Hz}, 2 \mathrm{H}), 7.27(\mathrm{~d}, J=8.7 \mathrm{~Hz}, 2 \mathrm{H}), 5.13(\mathrm{~d}, J=3.0 \mathrm{~Hz}, 1 \mathrm{H}), 4.22(\mathrm{q}, J=7.1$ $\mathrm{Hz}, 2 \mathrm{H}), 3.52(\mathrm{td}, J=10.4,3.6 \mathrm{~Hz}, 1 \mathrm{H}), 3.24(\mathrm{dd}, J=17.9,10.4 \mathrm{~Hz}, 1 \mathrm{H}), 2.94(\mathrm{dd}, J=$ 17.9, $3.9 \mathrm{~Hz}, 1 \mathrm{H}), 2.40(\mathrm{~s}, 3 \mathrm{H}), 1.23(\mathrm{t}, J=7.13 \mathrm{~Hz}, 3 \mathrm{H}) ;{ }^{13} \mathrm{C} \mathrm{NMR}(100 \mathrm{MHz}$, $\left.\mathrm{CDCl}_{3}\right) \delta 203.4,172.4,169.2,136.7,132.2,123.2,119.0,62.9,62.3,48.2,32.9,29.9$, 13.9 ; IR (KBr, cm $\left.{ }^{-1}\right) 2979,1748,1715,1489,1211$. MS (CI) $\mathrm{m} / z(\%): 373\left(\mathrm{M}+\mathrm{NH}_{3}\right.$, 40), $371\left(\mathrm{M}+\mathrm{NH}_{3}, 44\right), 356(\mathrm{M}+\mathrm{H}, 96), 354(\mathrm{M}+\mathrm{H}, 100), 276$ (33). HRMS: calcd for $\mathrm{C}_{15} \mathrm{H}_{16} \mathrm{BrNO}_{4}+\mathrm{H}, 356.0320$; found 356.0338.

trans- Ethyl 3-acetyl- $N$-(4-chlorophenyl)-5-oxopyrrolidine-2-carboxylate (trans-4g)<smiles>CCOC(=O)[C@H]1[C@@H](C(C)=O)CC(=O)N1c1ccc(Cl)cc1</smiles>

Lactam $4 \mathrm{~g}$ was obtained as a sticky solid $(24 \%) .{ }^{1} \mathrm{H}$ NMR $\left(400 \mathrm{MHz}, \mathrm{CDCl}_{3}\right) \delta 7.41(\mathrm{~d}$, $J=8.9 \mathrm{~Hz}, 2 \mathrm{H}), 7.34(\mathrm{~d}, J=8.9 \mathrm{~Hz}, 2 \mathrm{H}), 5.05(\mathrm{~d}, J=3.0 \mathrm{~Hz}, 1 \mathrm{H}), 4.19(\mathrm{q}, J=7.1 \mathrm{~Hz}$, $2 \mathrm{H}), 3.36(\mathrm{~m}, J=10.3,3.5 \mathrm{~Hz}, 1 \mathrm{H}), 3.03(\mathrm{dd}, J=17.3,10.3 \mathrm{~Hz}, 1 \mathrm{H}), 2.74(\mathrm{dd}, J=$ 17.3, $3.5 \mathrm{~Hz}, 1 \mathrm{H}), 2.33(\mathrm{~s}, 3 \mathrm{H}), 1.21(\mathrm{t}, J=7.12 \mathrm{~Hz}, 3 \mathrm{H}) .{ }^{13} \mathrm{C} \mathrm{NMR}\left(100 \mathrm{MHz}, \mathrm{CDCl}_{3}\right)$ $\delta 203.5,171.1,170.6,136.0,131.6,129.1,124.0,62.2,62.0,47.4,33.2,28.0,14.0$; IR $\left(\mathrm{KBr}, \mathrm{cm}^{-1}\right) 2979,1752,1716,1591,1492,1197 . \mathrm{MS}(\mathrm{CI}) \mathrm{m} / \mathrm{z}(\%): 327\left(\mathrm{M}+\mathrm{NH}_{3}, 36\right)$, $310(\mathrm{M}+\mathrm{H}, 100)$. HRMS: calcd for $\mathrm{C}_{15} \mathrm{H}_{16} \mathrm{ClNO}_{4}+\mathrm{H}, 310.0846$; found 310.0862 . 
<smiles>CCOC(=O)c1ccc(N2C(=O)CC[C@@H](C(C)=O)[C@H]2C(=O)OCC)cc1</smiles>

Lactam 4h was obtained as a sticky solid (45\%). ${ }^{1} \mathrm{H}$ NMR $\left(400 \mathrm{MHz}, \mathrm{CDCl}_{3}\right) \delta 8.07$ (d, $J=8.6 \mathrm{~Hz}, 2 \mathrm{H}), 7.48(\mathrm{~d}, J=8.6 \mathrm{~Hz}, 2 \mathrm{H}), 4.97(\mathrm{dd}, J=2.2,0.7 \mathrm{~Hz}, 1 \mathrm{H}), 4.37(\mathrm{~m}, 2 \mathrm{H})$, $4.18(\mathrm{~m}, 2 \mathrm{H}), 3.29(\mathrm{~m}, J=5.7,5.6,2.3 \mathrm{~Hz}, 1 \mathrm{H}), 2.62(\mathrm{~m}, J=5.7,5.6,2.3 \mathrm{~Hz}, 1 \mathrm{H}), 2.45$ $(\mathrm{m}, 1 \mathrm{H}), 2.33(\mathrm{~s}, 3 \mathrm{H}), 2.29(\mathrm{~m}, 2 \mathrm{H}), 1.38(\mathrm{t}, J=7.1 \mathrm{~Hz}, 3 \mathrm{H}), 1.23(\mathrm{t}, J=7.1 \mathrm{~Hz}, 3 \mathrm{H})$. ${ }^{13} \mathrm{C}$ NMR $\left(100 \mathrm{MHz}, \mathrm{CDCl}_{3}\right) \delta 205.6,171.2,169.5,166.1,146.3,130.7,129.5,127.6$, $62.4,62.4,61.2,49.1,29.9,27.4,21.1,14.5,14.2$. IR $\left(\mathrm{KBr}, \mathrm{cm}^{-1}\right) 2979,1716,1671$, 1600, 1394, 1179. MS (EI) $\mathrm{m} / \mathrm{z}(\%): 361$ (M, 11), 288 (36), 246 (100). HRMS: calcd for $\mathrm{C}_{19} \mathrm{H}_{23} \mathrm{NO}_{6}, 361.1525$; found 361.1497 .

trans-Ethyl 3-acetyl- $N$-(3-nitrophenyl)-6-oxopiperidine-2-carboxylate (trans-4i)<smiles>CCOC(=O)[C@H]1[C@@H](C(C)=O)CCC(=O)N1c1cccc([N+](=O)[O-])c1</smiles>

Lactam 4i was obtained as a sticky solid (40\%). ${ }^{1} \mathrm{H}$ NMR $\left(400 \mathrm{MHz}, \mathrm{CDCl}_{3}\right) \delta 8.32(\mathrm{t}, J$ $=2.1 \mathrm{~Hz}, 1 \mathrm{H}), 8.16(\mathrm{~m}, J=8.3,2.1,0.9 \mathrm{~Hz}, 1 \mathrm{H}), 8.16(\mathrm{~m}, J=8.3,2.1,0.9 \mathrm{~Hz}, 1 \mathrm{H})$, $7.58(\mathrm{~m}, J=8.1,8.1 \mathrm{~Hz}, 1 \mathrm{H}), 4.97(\mathrm{~d}, J=1.1 \mathrm{~Hz}, 1 \mathrm{H}), 4.21(\mathrm{q}, J=7.1 \mathrm{~Hz}, 2 \mathrm{H}), 3.36$ $(\mathrm{m}, J=5.4,5.2,1.8 \mathrm{~Hz}, 1 \mathrm{H}), 2.63(\mathrm{~m}, J=17.4,5.9,5.9 \mathrm{~Hz}, 1 \mathrm{H}), 2.35(\mathrm{~s}, 3 \mathrm{H}), 2.32(\mathrm{~m}$, $3 \mathrm{H}), 1.26(\mathrm{t}, J=7.1 \mathrm{~Hz}, 3 \mathrm{H}) .{ }^{13} \mathrm{C} \mathrm{NMR}\left(100 \mathrm{MHz}, \mathrm{CDCl}_{3}\right) \delta 205.2,170.9,169.2$, 148.6, 143.0, 134.0, 129.8, 123.2, 122.4, 62.5, 62.2, 48.9, 29.7, 29.2, 21.1, 14.1; IR $\left(\mathrm{KBr}, \mathrm{cm}^{-1}\right)$ 2979, 1743, 1707, 1680, 1519, 1340, 1035. MS (EI) $\mathrm{m} / \mathrm{z}(\%): 334(\mathrm{M}, 4)$, 261 (23), 219 (100). HRMS: calcd for $\mathrm{C}_{16} \mathrm{H}_{18} \mathrm{~N}_{2} \mathrm{O}_{6}, 334.1165$; found 334. 1176. 


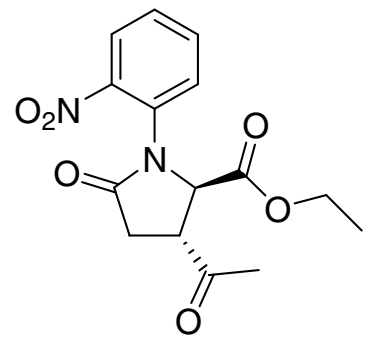

Lactam 4j was obtained as a sticky solid (11\%). ${ }^{1} \mathrm{H}$ NMR (400 MHz, $\left.\mathrm{CDCl}_{3}\right) \delta 8.06$ $(\mathrm{dd}, J=8.1,1.4 \mathrm{~Hz}, 1 \mathrm{H}), 7.67(\mathrm{~m}, J=7.9,7.5,1.5 \mathrm{~Hz}, 1 \mathrm{H}), 7.52(\mathrm{~m}, 2 \mathrm{H}), 5.06(\mathrm{~d}, J=$ $4.1 \mathrm{~Hz}, 1 \mathrm{H}), 4.15(\mathrm{~m}, 2 \mathrm{H}), 3.60(\mathrm{~m}, J=10.1,5.3,4.1 \mathrm{~Hz}, 1 \mathrm{H}), 2.97(\mathrm{dd}, J=17.3,10.1$ $\mathrm{Hz}, 1 \mathrm{H}), 2.81(\mathrm{dd}, J=17.3,5.3 \mathrm{~Hz}, 1 \mathrm{H}), 2.37(\mathrm{~s}, 3 \mathrm{H}), 1.15(\mathrm{t}, J=7.1 \mathrm{~Hz}, 3 \mathrm{H}) .{ }^{13} \mathrm{C}$ NMR $\left(100 \mathrm{MHz}, \mathrm{CDCl}_{3}\right) \delta 203.7,172.3,169.9,134.3,131.1,130.0,129.1,126.1$, 125.9, 63.1, 62.5, 48.2, 32.3, 28.4, 14.1; IR (KBr, cm $\left.{ }^{-1}\right)$ 2979, 1716, 1600, 1537, 1367, 1017. MS (EI) m/z (\%): 320 (M, 5), 290 (5), 247 (38), 205 (100), 177 (82). HRMS: calcd for $\mathrm{C}_{15} \mathrm{H}_{16} \mathrm{~N}_{2} \mathrm{O}_{6}, 320.1008$; found 320.1024 .

\section{Ethyl 6-methylquinoline-2-carboxylate (5a)}<smiles>CCOC(=O)c1ccc2cc(C)ccc2n1</smiles>

Product 5a was obtained as a thick oil $(10 \%) .{ }^{1} \mathrm{H}$ NMR $\left(400 \mathrm{MHz}, \mathrm{CDCl}_{3}\right) \delta 8.16(\mathrm{~m}$, $3 \mathrm{H}), 7.60(\mathrm{~m}, 2 \mathrm{H}), 4.54(\mathrm{q}, J=7.12 \mathrm{~Hz}, 2 \mathrm{H}), 2.55$ (s, 3H), 1.48 (t, $J=7.13 \mathrm{~Hz}, 3 \mathrm{H})$; ${ }^{13} \mathrm{C}$ NMR $\left(100 \mathrm{MHz}, \mathrm{CDCl}_{3}\right) \delta 165.60,147.34,146.18,138.81,136.35,132.55,130.38$, 129.35, 126.25, 121.04, 62.11, 21.74, 14.34; IR (KBr, cm $\left.{ }^{-1}\right)$ 2970, 1734, 1707, 1466, 1143; MS (EI) m/z (\%): 215 (M, 6), 171 (14), 143 (100); HRMS: calcd for $\mathrm{C}_{13} \mathrm{H}_{13} \mathrm{NO}_{2}$, 215, 0946; found 215.0968 .

\section{Ethyl 6-methoxyquinoline-2-carboxylate (5b)}

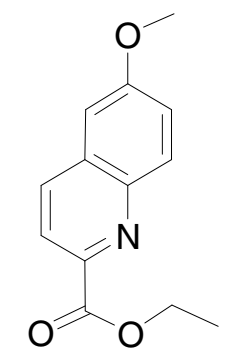

Quinoline 5b was obtained as a solid (31\%). ${ }^{1} \mathrm{H}$ NMR $\left(400 \mathrm{MHz}, \mathrm{CDCl}_{3}\right) \delta 8.20(\mathrm{~d}, J=$ $9.3 \mathrm{~Hz}, 1 \mathrm{H}), 8.15$ (bs, 2H), 7.43 (dd, $J=9.3,2.8 \mathrm{~Hz}, 1 \mathrm{H}), 7.10(\mathrm{~d}, J=2.8 \mathrm{~Hz}, 1 \mathrm{H}), 4.55$ 
$(\mathrm{q}, J=7.1 \mathrm{~Hz}, 2 \mathrm{H}), 3.96(\mathrm{~s}, 3 \mathrm{H}), 1.49(\mathrm{t}, J=7.1 \mathrm{~Hz}, 3 \mathrm{H}) .{ }^{13} \mathrm{C} \mathrm{NMR}\left(100 \mathrm{MHz}, \mathrm{CDCl}_{3}\right)$ $\delta 165.7,159.6,146.0,143.9,135.8,132.4,130.9,123.6,121.7,104.8,62.2,55.8,14.6$ IR (KBr, cm $\left.{ }^{-1}\right)$ 2970, 1725, 1618, 1429, 1098. MS (EI) m/z (\%): 231 (M, 17), 159 (100), 134 (20).

\section{Ethyl 4-oxo-2-( $N$-p-tolylacetamido)pentanoate (6a)}<smiles>CCOC(=O)C(CC(C)=O)N(C(C)=O)c1ccc(C)cc1</smiles>

Mannich adduct 6a was obtained as a thick oil (20\%). ${ }^{1} \mathrm{H}$ NMR $\left(400 \mathrm{MHz}, \mathrm{CDCl}_{3}\right) \delta$ $7.20(J=8.3 \mathrm{~Hz}, 2 \mathrm{H}), 7.16(J=8.3 \mathrm{~Hz}, 2 \mathrm{H}), 4.96(\mathrm{~m}, 1 \mathrm{H}), 4.21(\mathrm{q}, J=7.1 \mathrm{~Hz}, 2 \mathrm{H})$, $3.44(\mathrm{dd}, J=17.6,7.0 \mathrm{~Hz}, 1 \mathrm{H}), 2.78(\mathrm{dd}, J=17.6,6.2 \mathrm{~Hz}, 1 \mathrm{H}), 2.38(\mathrm{~s}, 3 \mathrm{H}), 2.14(\mathrm{~s}$, $3 \mathrm{H}), 1.82(\mathrm{~s}, 3 \mathrm{H}), 1.29(\mathrm{t}, J=7.1 \mathrm{~Hz}, 3 \mathrm{H}) .{ }^{13} \mathrm{C} \mathrm{NMR}\left(100 \mathrm{MHz}, \mathrm{CDCl}_{3}\right) \delta 205.5,171.2$, 170.6, 140.1, 138.7, 130.5, 128.3, 61.8, 58.5, 43.6, 30.4, 23.0, 21.3, 14.3. IR $\left(\mathrm{KBr}, \mathrm{cm}^{-1}\right)$ 2979, 1734, 1716, 1662, 1510, 1492, 1367. MS (EI) m/z (\%): 291 (M, 8), 249 (37), 176 (100), 134 (56). HRMS: calcd for $\mathrm{C}_{16} \mathrm{H}_{21} \mathrm{NO}_{4}, 291.1471$; found 291.1495.

Ethyl 4-[N-(1-ethoxy-1,4-dioxopentan-2-yl)acetamido]benzoate (6b)<smiles>CCOC(=O)c1ccc(N(C(C)=O)C(CC(C)=O)C(=O)OCC)cc1</smiles>

Mannich adduct $\mathbf{6 b}$ was obtained as a thick oil $(9 \%) .{ }^{1} \mathrm{H}$ NMR $\left(400 \mathrm{MHz}, \mathrm{CDCl}_{3}\right) \delta$ $8.10(\mathrm{~d}, J=8.5 \mathrm{~Hz}, 2 \mathrm{H}), 7.39(\mathrm{~d}, J=8.5 \mathrm{~Hz}, 2 \mathrm{H}), 4.89(\mathrm{t}, J=6.5 \mathrm{~Hz}, 1 \mathrm{H}), 4.40(\mathrm{q}, J=$ $7.1 \mathrm{~Hz}, 2 \mathrm{H}), 4.23(\mathrm{q}, J=7.1 \mathrm{~Hz}, 2 \mathrm{H}), 3.50(\mathrm{dd}, J=17.9,6.5 \mathrm{~Hz}, 1 \mathrm{H}), 2.89$ (dd, $J=$ $17.9,6.5 \mathrm{~Hz}, 1 \mathrm{H}), 2.16(\mathrm{~s}, 3 \mathrm{H}), 1.85(\mathrm{~s}, 3 \mathrm{H}), 1.41(\mathrm{t}, J=7.13 \mathrm{~Hz}, 3 \mathrm{H}), 1.30(\mathrm{t}, J=7.14$ $\mathrm{Hz}, 3 \mathrm{H}) .{ }^{13} \mathrm{C}$ NMR $\left(100 \mathrm{MHz}, \mathrm{CDCl}_{3}\right) \delta 205.1,170.1,165.5,146.6,131.0,130.7$, 130.4, 128.2, 61.7, 61.3, 58.6, 43.5, 30.1, 22.8, 14.3, 14.1; IR (KBr, cm $\left.{ }^{-1}\right) 2907,1716$, 1600, 1304, 1107. MS (CI) m/z (\%): 350 (M+H, 74), 225 (100), 208 (99); HRMS: calcd for $\mathrm{C}_{18} \mathrm{H}_{23} \mathrm{NO}_{6}+\mathrm{H}, 350.1604$; found 350.1578 . 


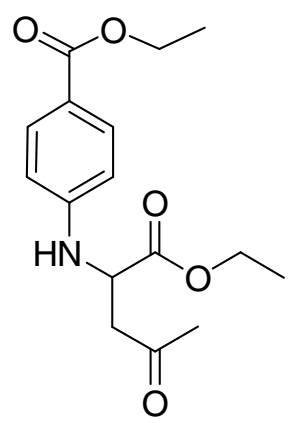

Mannich adduct 7b was obtained as a thick oil (33\%). ${ }^{1} \mathrm{H}$ NMR $\left(400 \mathrm{MHz}, \mathrm{CDCl}_{3}\right) \delta$ $7.87(\mathrm{~d}, J=8.9 \mathrm{~Hz}, 2 \mathrm{H}), 6.60(\mathrm{~d}, J=8.7 \mathrm{~Hz}, 2 \mathrm{H}), 4.93(\mathrm{br} \mathrm{d}, J=7.6 \mathrm{~Hz}, 1 \mathrm{H}), 4.46(\mathrm{~m}$, $1 \mathrm{H}), 4.31(\mathrm{~m}, 2 \mathrm{H}), 4.21(\mathrm{~m}, 2 \mathrm{H}), 3.05(\mathrm{t}, J=5.23 \mathrm{~Hz}, 2 \mathrm{H}), 2.18(\mathrm{~s}, 3 \mathrm{H}), 1.36(\mathrm{~m}, 3 \mathrm{H})$, $1.25(\mathrm{~m}, 3 \mathrm{H}) .{ }^{13} \mathrm{C}$ NMR $\left(100 \mathrm{MHz}, \mathrm{CDCl}_{3}\right) \delta 205.5,171.8,166.5,150.0,131.3,119.6$, 112.0, 61.5, 60.1, 51.6, 44.9, 30.0, 14.2, 13.9. IR (KBr, $\left.\mathrm{cm}^{-1}\right) 3337,2979,1707,1671$, 1600, 1268, 1170. MS (CI) $\mathrm{m} / z(\%): 308(\mathrm{M}+\mathrm{H})$. HRMS: calcd for $\mathrm{C}_{16} \mathrm{H}_{21} \mathrm{NO}_{5}+\mathrm{H}$, 308.1498; found 308.1472.

Ethyl 2-(4-nitrophenylamino)-4-oxopentanoate (7c)<smiles>CCOC(=O)C(CC(C)=O)Nc1ccc([N+](=O)[O-])cc1</smiles>

Mannich adduct 7c was obtained as a thick oil (74\%). ${ }^{1} \mathrm{H}$ NMR $\left(400 \mathrm{MHz}, \mathrm{CDCl}_{3}\right) \delta$ $8.07(\mathrm{~d}, J=9.2 \mathrm{~Hz}, 2 \mathrm{H}), 6.61(\mathrm{~d}, J=9.2 \mathrm{~Hz}, 2 \mathrm{H}), 5.41(\mathrm{~s}, 1 \mathrm{H}), 4.49(\mathrm{t}, J=4.9 \mathrm{~Hz}, 1 \mathrm{H})$, $4.22(\mathrm{~m}, 2 \mathrm{H}), 3.10(\mathrm{~m}, 2 \mathrm{H}), 2.21(\mathrm{~s}, 3 \mathrm{H}), 1.26(\mathrm{t}, J=7.1 \mathrm{~Hz}, 3 \mathrm{H}) .{ }^{13} \mathrm{C} \mathrm{NMR}(100 \mathrm{MHz}$, $\left.\mathrm{CDCl}_{3}\right) \delta \quad 205.8,171.5,152.0,139.0,126.5,112.0,62.3,51.8,45.1,30.5,14.3$. IR $\left(\mathrm{KBr}, \mathrm{cm}^{-1}\right) 3373,2988,1734,1707,1591,1501,1322,1107 ; \mathrm{MS}(\mathrm{EI}) \mathrm{m} / \mathrm{z}(\%): 280(\mathrm{M}$, 14), 207 (95), 165 (100); HRMS: calcd for $\mathrm{C}_{13} \mathrm{H}_{16} \mathrm{~N}_{2} \mathrm{O}_{5}, 280.1059$; found 280.1073.

\section{4-(4-Nitrophenyl)-4-(4-nitrophenylamino)butan-2-one (7d)}<smiles>CC(=O)CC(Nc1ccc([N+](=O)[O-])cc1)c1ccc([N+](=O)[O-])cc1</smiles> 
Mannich adduct 7d was obtained as a thick oil $(35 \%) .{ }^{1} \mathrm{H}$ NMR $\left(400 \mathrm{MHz}, \mathrm{CDCl}_{3}\right) \delta$ $8.20(\mathrm{~d}, J=8.7 \mathrm{~Hz}, 2 \mathrm{H}), 8.00(\mathrm{~d}, J=9.2 \mathrm{~Hz}, 2 \mathrm{H}), 7.54(\mathrm{~d}, J=8.7 \mathrm{~Hz}, 2 \mathrm{H}), 6.48(\mathrm{~d}, J=$ $9.2 \mathrm{~Hz}, 2 \mathrm{H}), 5.03(\mathrm{t}, J=5.8 \mathrm{~Hz}, 1 \mathrm{H}), 3.09(\mathrm{~m}, 2 \mathrm{H}), 2.16(\mathrm{~s}, 3 \mathrm{H}) .{ }^{13} \mathrm{C} \mathrm{NMR}(100 \mathrm{MHz}$, $\left.\mathrm{CDCl}_{3}\right) \delta 206.0,151.8,148.7,147.7,139.2,127.5,126.4,124.5,112.4,53.5,49.8,31.2$. IR $\left(\mathrm{KBr}, \mathrm{cm}^{-1}\right)$ 3364, 2916, 1716, 1591, 1510, 1313, 1107; MS (EI) $\mathrm{m} / z$ (\%): 329 (M), 272 (100), 226 (25); HRMS: calcd for $\mathrm{C}_{16} \mathrm{H}_{15} \mathrm{~N}_{3} \mathrm{O}_{5}, 329.1012$; found 329.1037.

\section{4-(4-Chlorophenyl)-4-(4-nitrophenylamino)butan-2-one (7e)}<smiles>CC(=O)CC(Nc1ccc([N+](=O)[O-])cc1)c1ccc(Cl)cc1</smiles>

Mannich adduct 7e was obtained as a thick oil (63\%). ${ }^{1} \mathrm{H}$ NMR $\left(400 \mathrm{MHz}, \mathrm{CDCl}_{3}\right) \delta$ $7.99(\mathrm{~d}, J=9.1 \mathrm{~Hz}, 2 \mathrm{H}), 7.28(\mathrm{~d}, J=6.5 \mathrm{~Hz}, 4 \mathrm{H}), 6.47(\mathrm{~d}, J=6.1 \mathrm{~Hz}, 2 \mathrm{H}), 5.60(\mathrm{~s}, 1 \mathrm{H})$, $4.90(\mathrm{br} \mathrm{d}, J=5.1 \mathrm{~Hz}, 1 \mathrm{H}), 3.00(\mathrm{~m}, 2 \mathrm{H}), 2.12(\mathrm{~s}, 3 \mathrm{H}) .{ }^{13} \mathrm{C} \mathrm{NMR}\left(100 \mathrm{MHz}, \mathrm{CDCl}_{3}\right) \delta$ 206.7, 152.1, 139.5, 138.9, 133.9, 129.5, 127.8, 126.4, 112.4, 53.5, 50.1, 31.4. IR (KBr, $\left.\mathrm{cm}^{-1}\right)$ 3364, 2907, 1716, 1600, 1501, 1304, 1107. MS ((EI) m/z (\%): 318 (M, 16), 261 (100), 215 (20), 138 (19). HRMS: calcd for $\mathrm{C}_{16} \mathrm{H}_{15} \mathrm{ClN}_{2} \mathrm{O}_{3}, 318.0771$; found 318.0793.

\section{4-(4-Chlorophenyl)-4-(3,5-dinitrophenylamino)butan-2-one (7f)}<smiles>CC(=O)CC(Nc1cc([N+](=O)[O-])cc([N+](=O)[O-])c1)c1ccc(Cl)cc1</smiles>

Mannich adduct 7f was obtained as a thick oil (76\%). ${ }^{1} \mathrm{H}$ NMR $(400 \mathrm{MHz}, \mathrm{MeOD}) \delta$ $8.20(\mathrm{dd}, J=4.4,2.0 \mathrm{~Hz}, 1 \mathrm{H}), 7.63(\mathrm{~m}, J=2.0 \mathrm{~Hz}, 2 \mathrm{H}), 7.33(\mathrm{~m}, 4 \mathrm{H}), 4.92(\mathrm{~m}, 1 \mathrm{H})$, 3.08 (ddd, $J=17.1,8.0,1.9 \mathrm{~Hz}, 1 \mathrm{H}), 2.93(\mathrm{ddd}, J=17.1,4.8,2.0 \mathrm{~Hz}, 1 \mathrm{H}), 2.18(\mathrm{~s}, 3 \mathrm{H})$ ${ }^{13} \mathrm{C}$ NMR (100 MHz, MeOD) $\delta$ 206.6, 149.3, 149.0, 139.4, 133.8, 129.4, 127.8, 112.4, 106.3, 53.2, 50.6, 30.6. IR (KBr, cm $\left.{ }^{-1}\right) 3382,3095,1698,1537,1340,1089$. MS (EI) $m / z$ (\%): 363 (M, 17), 346 (17), 306 (100); HRMS: calcd for $\mathrm{C}_{16} \mathrm{H}_{14} \mathrm{ClN}_{3} \mathrm{O}_{5}, 363.0622$; found 363.0648 . 
trans-Ethyl 3-acetyl-6-oxo- $N$-p-tolylpiperidine-2-carboxylate (trans-4a)<smiles>CCOC(=O)[C@H]1[C@@H](C(C)=O)CCC(=O)N1c1ccc(C)cc1</smiles>

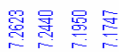

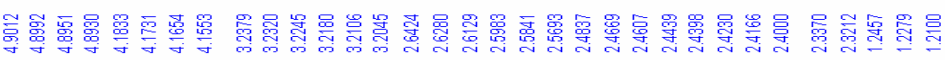

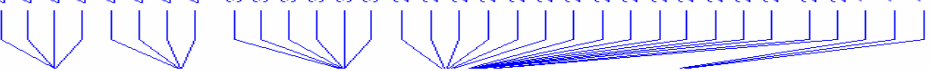

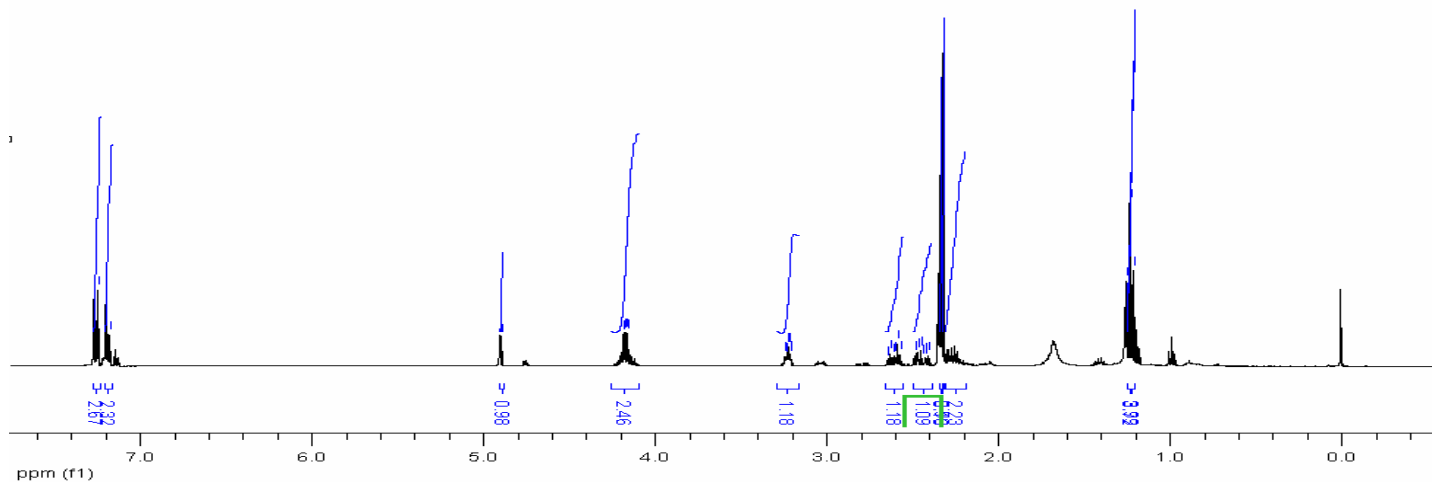

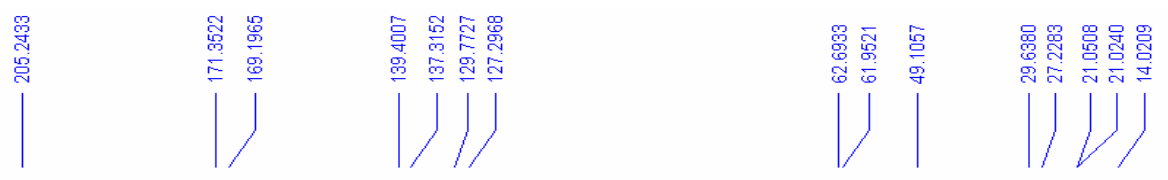

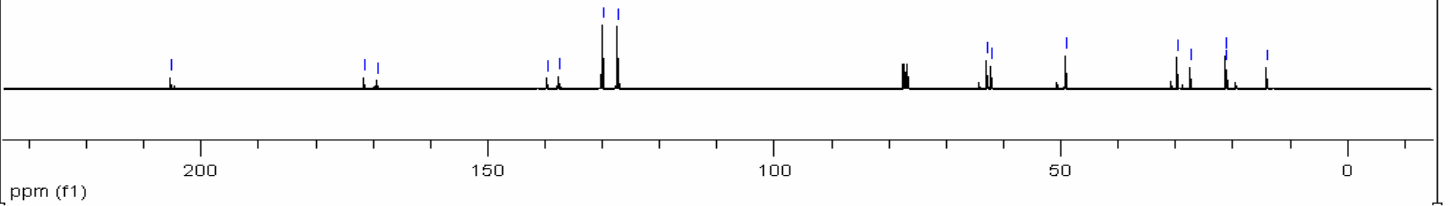


trans-Ethyl 3-acetyl-5-oxo- $N$-p-tolylpyrrolidine-2-carboxylate (trans-4b)
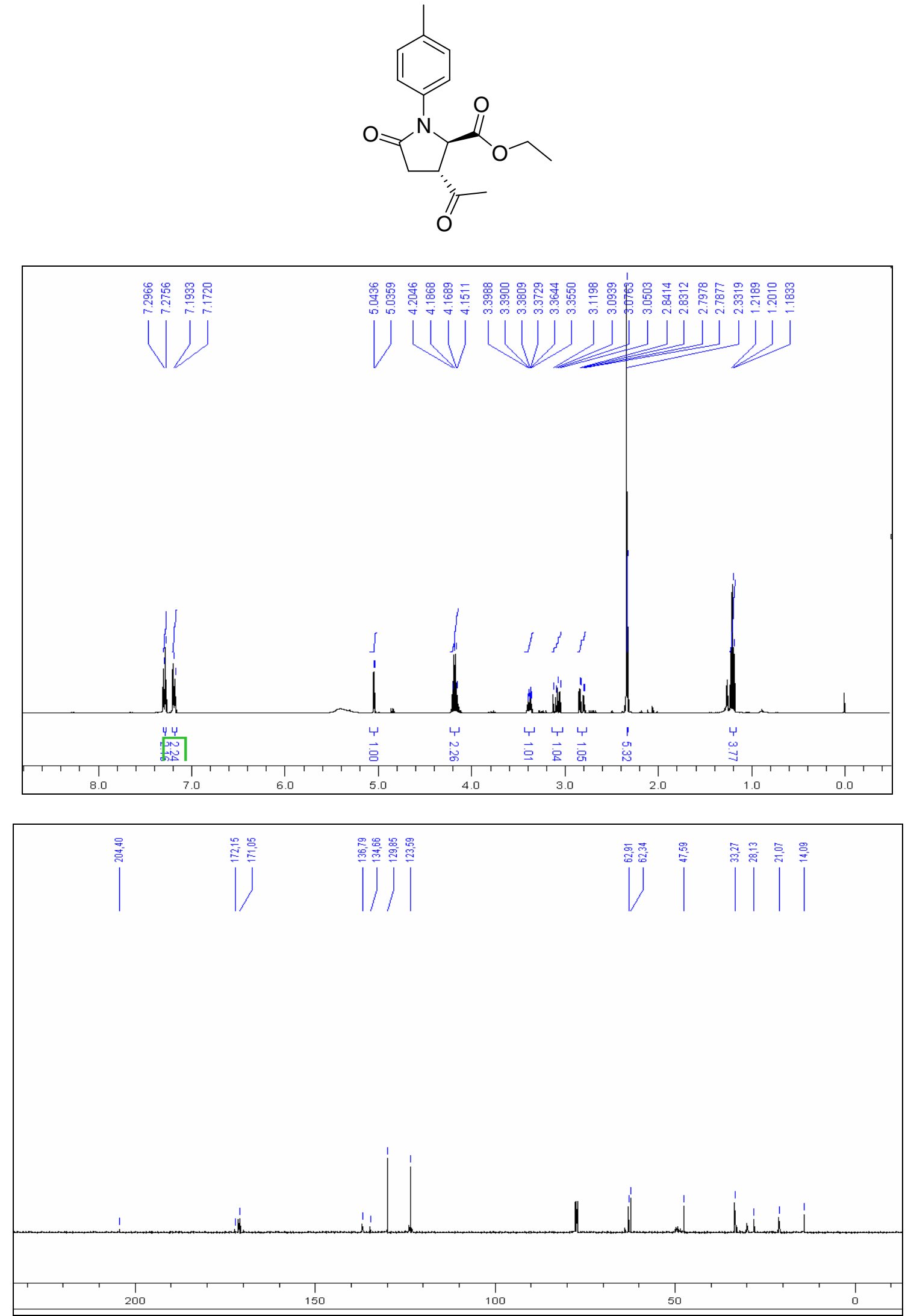
cis-Ethyl 3-acetyl- $N$-(4-methoxyphenyl)-6-oxopiperidine-2-carboxylate (cis-4c)<smiles>CCOC(=O)[C@H]1[C@@H](C(C)=O)CCC(=O)N1c1ccc(OC)cc1</smiles>
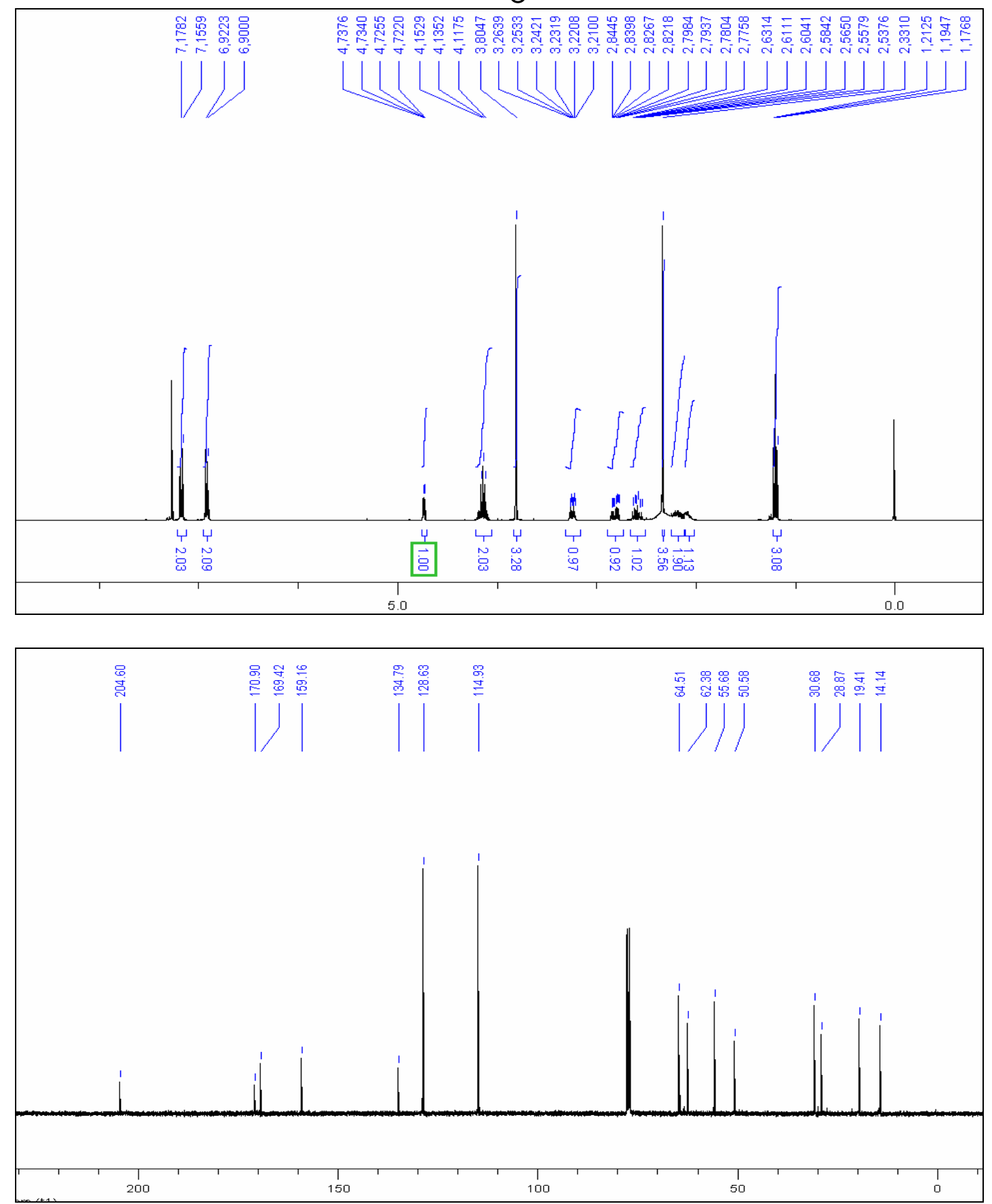
trans-Ethyl 3-acetyl- $N$-(4-methoxyphenyl)-6-oxopiperidine-2-carboxylate (trans-4c)<smiles>CCOC(=O)[C@H]1[C@@H](C(C)=O)CCC(=O)N1c1ccc(OC)cc1</smiles>
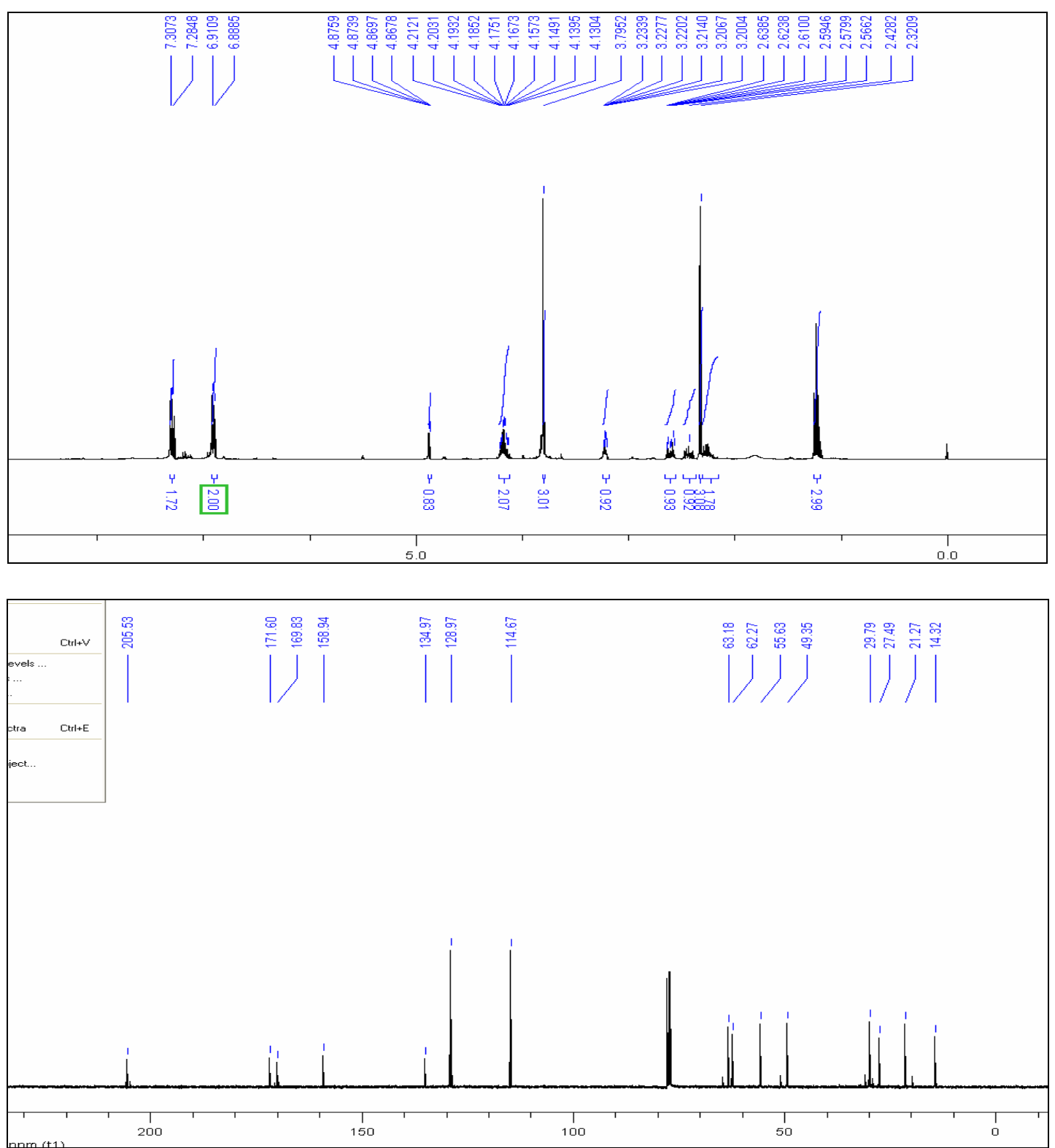
trans-Ethyl 3-acetyl- $N$-(4-methoxyphenyl)-5-oxopyrrolidine-2-carboxylate (trans-4d)
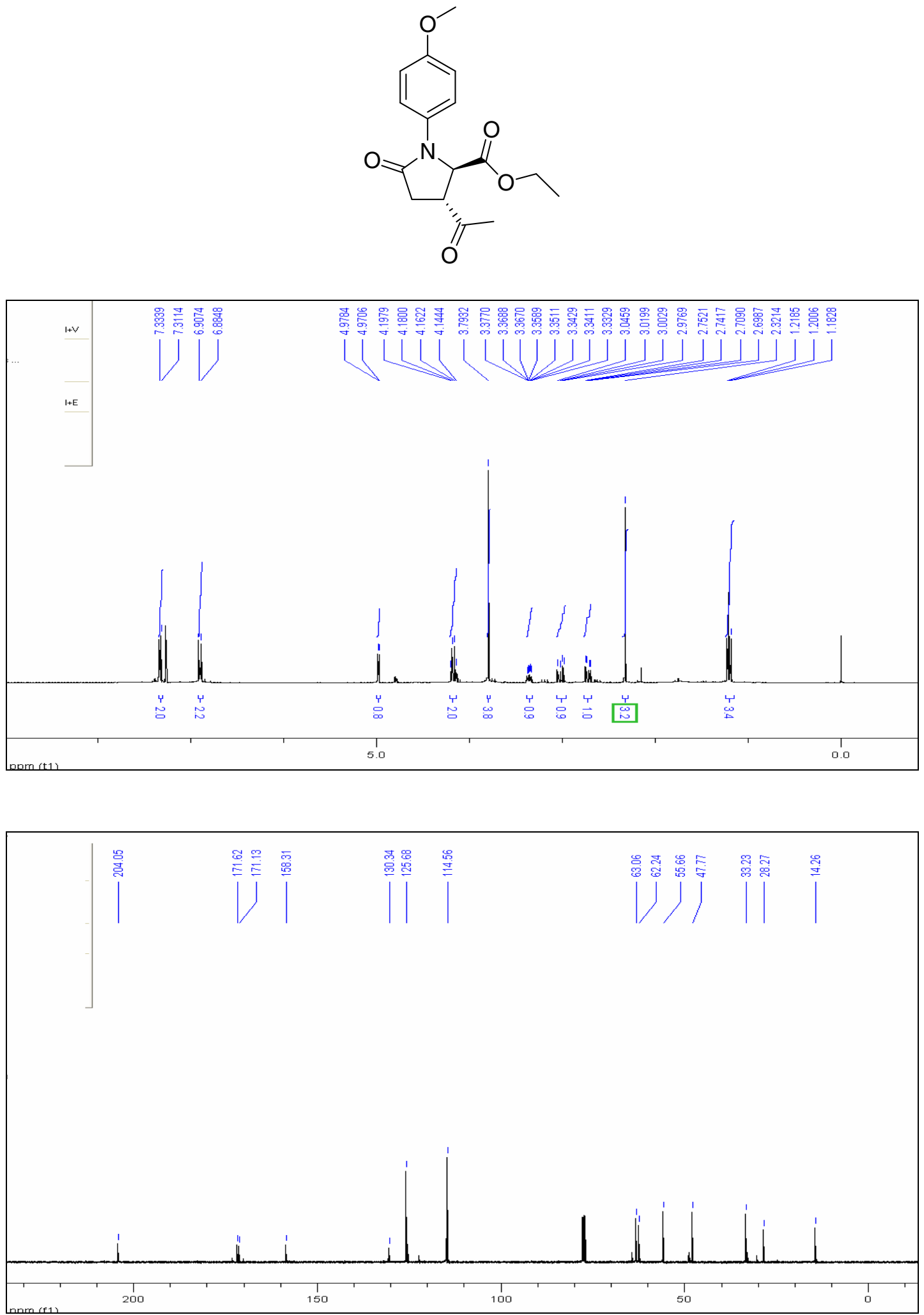
trans-Ethyl 3-acetyl- $N$-(4-bromophenyl)-6-oxopiperidine-2-carboxylate (trans-4e)
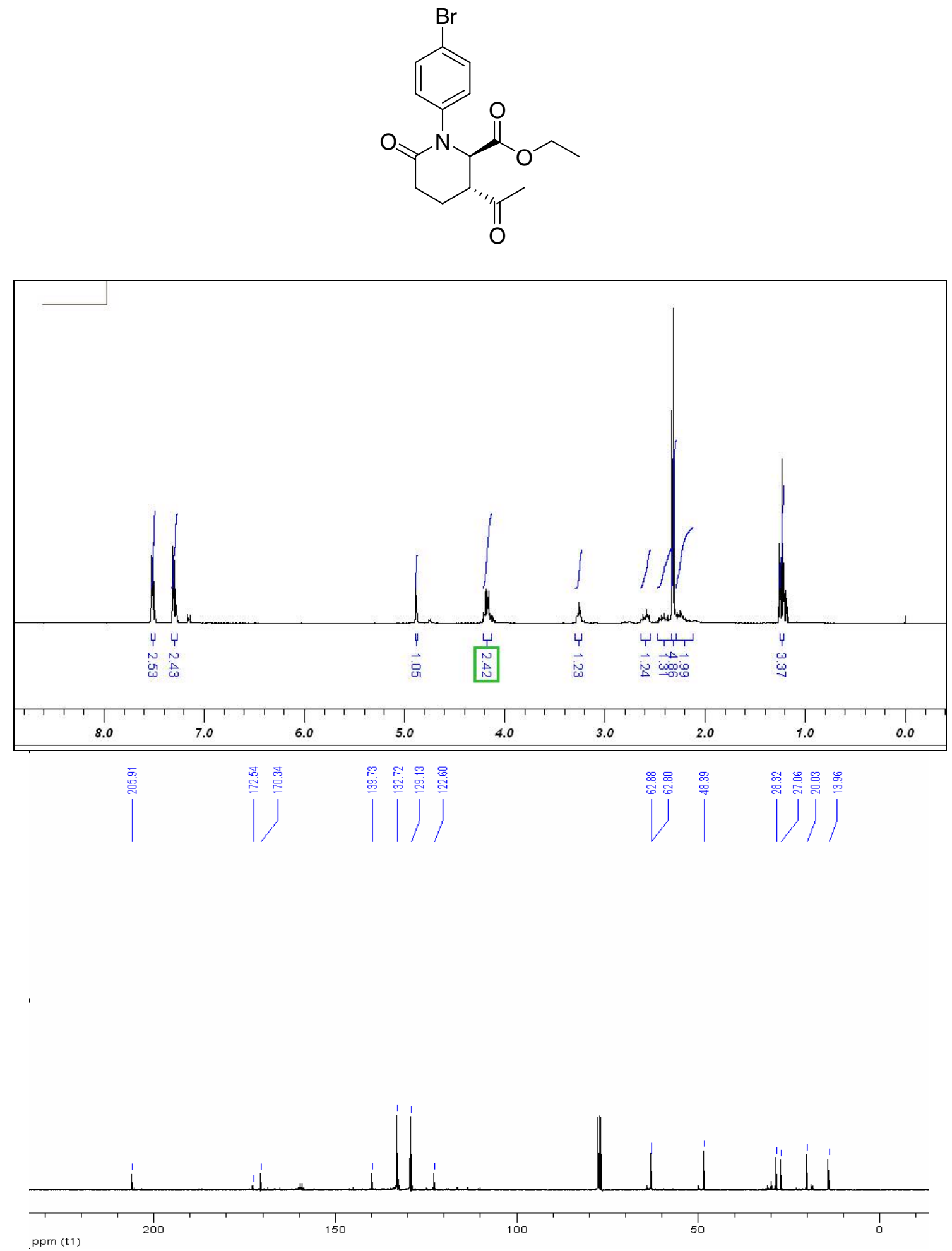
trans-Ethyl 3-acetyl- $N$-(4-bromophenyl)-5-oxopyrrolidine-2-carboxylate (trans-4f)<smiles>CCOC(=O)[C@H]1[C@@H](C(C)=O)CC(=O)N1c1ccc(Br)cc1</smiles>
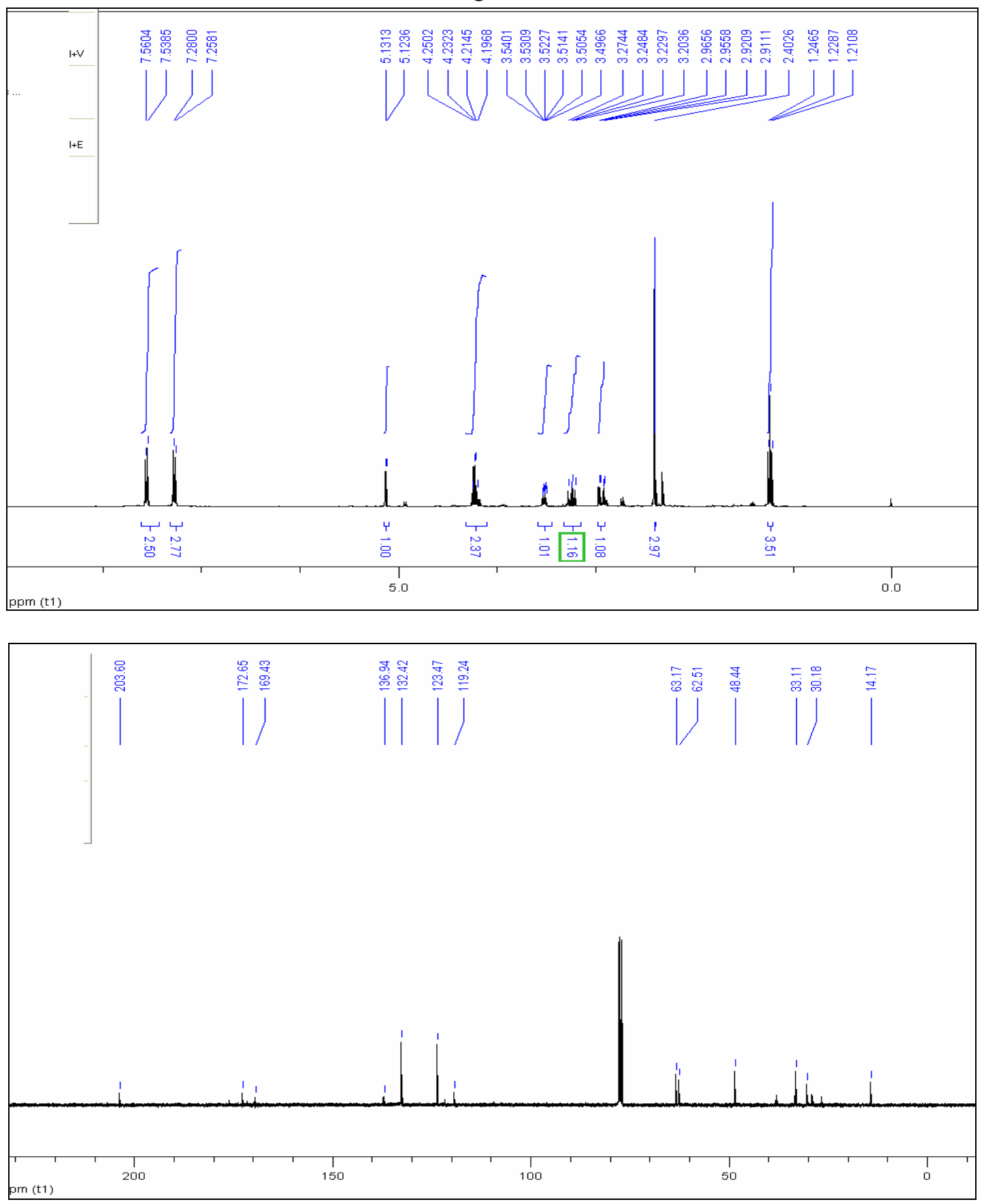
trans-Ethyl 3-acetyl- $N$-(4-chlorophenyl)-5-oxopyrrolidine-2-carboxylate (trans-4g)<smiles>CCOC(=O)[C@@H]1[C@H](C(C)=O)CC(=O)N1c1ccc(Cl)cc1</smiles>
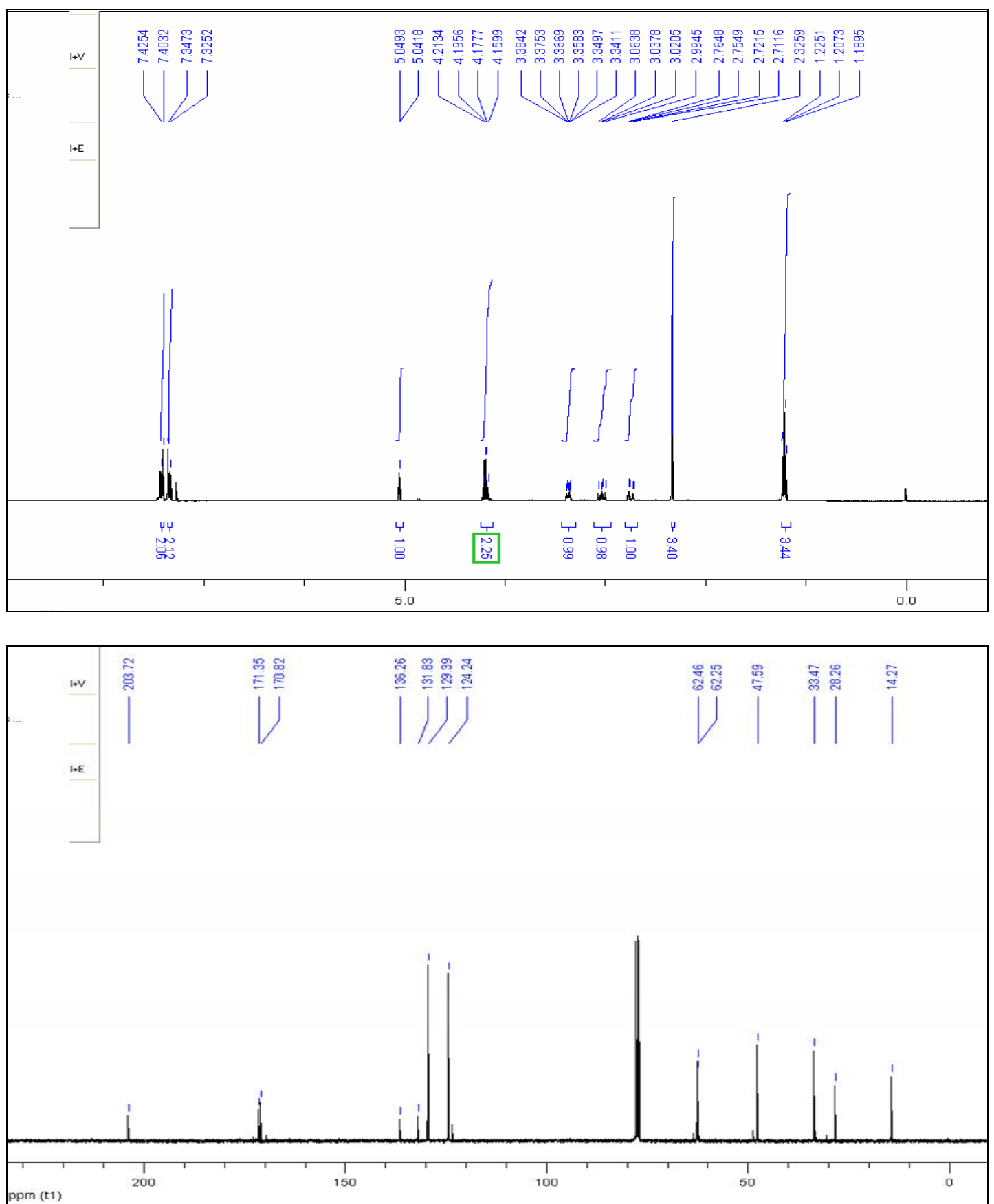

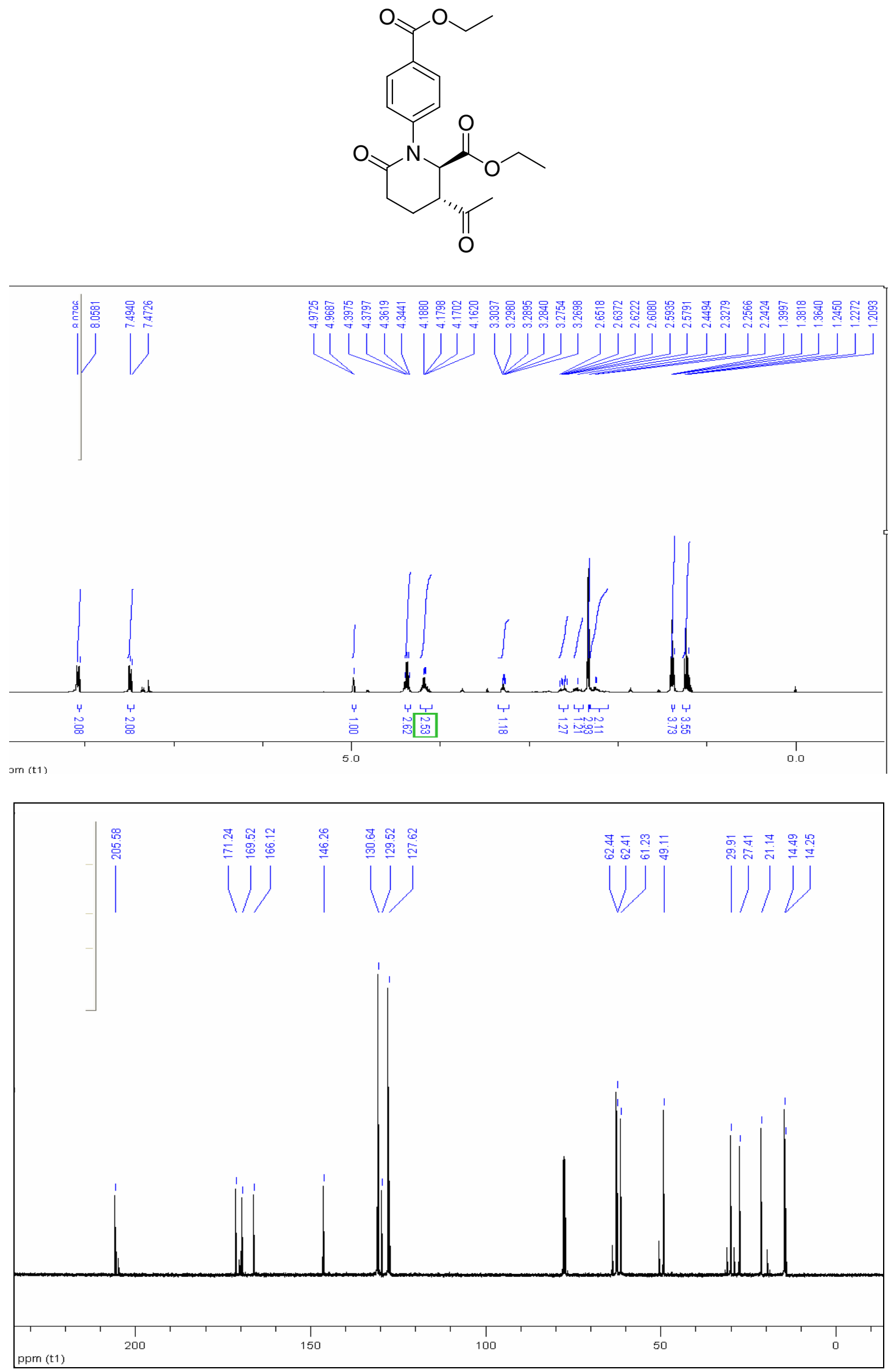
trans-Ethyl 3-acetyl- $N$-(3-nitrophenyl)-6-oxopiperidine-2-carboxylate (trans-4i)<smiles>CCOC(=O)[C@H]1[C@@H](C(C)=O)CCC(=O)N1c1cccc([N+](=O)[O-])c1</smiles>
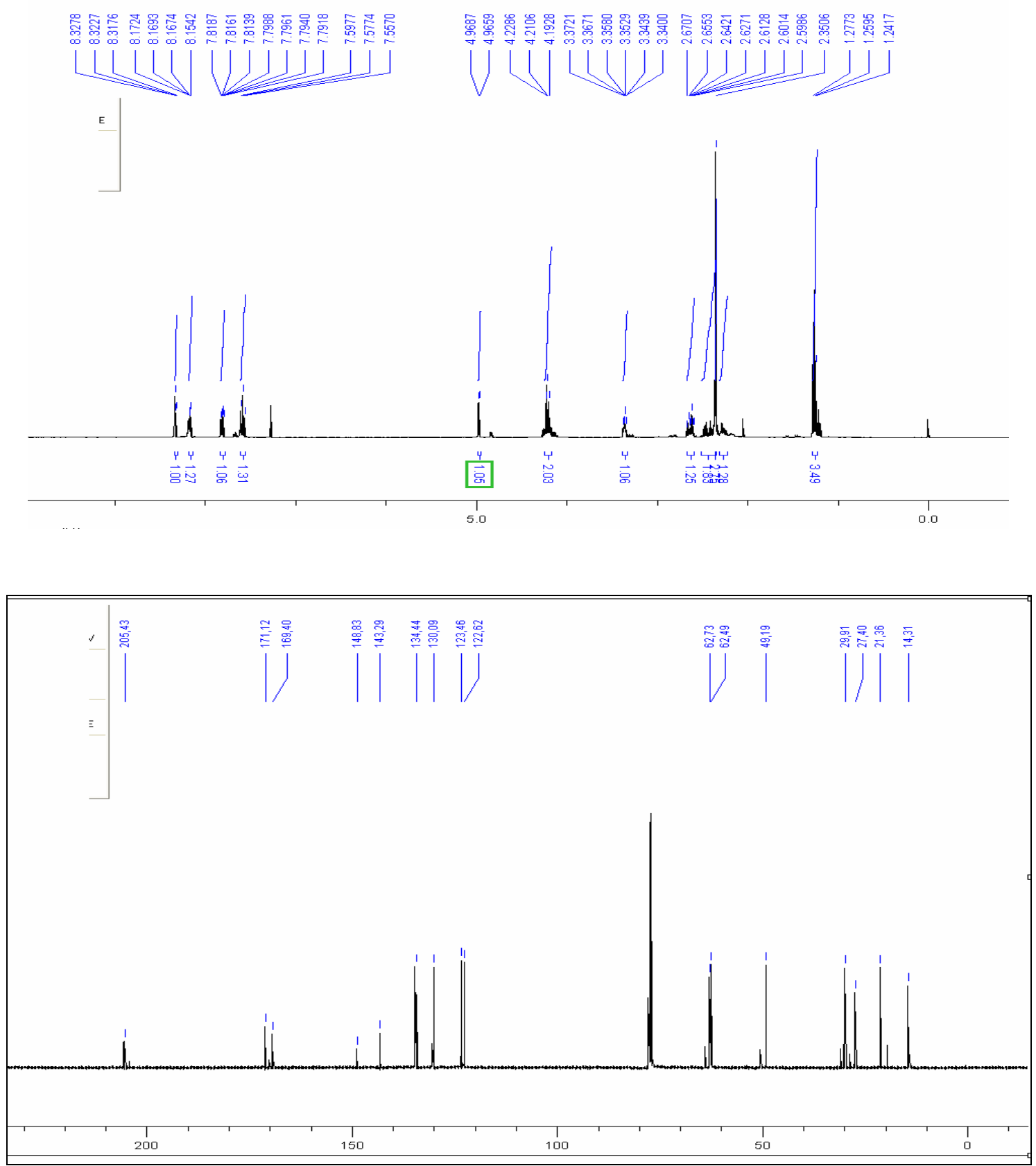
trans-Ethyl 3-acetyl- $N$-(2-nitrophenyl)-5-oxopyrrolidine-2-carboxylate (trans-4j)
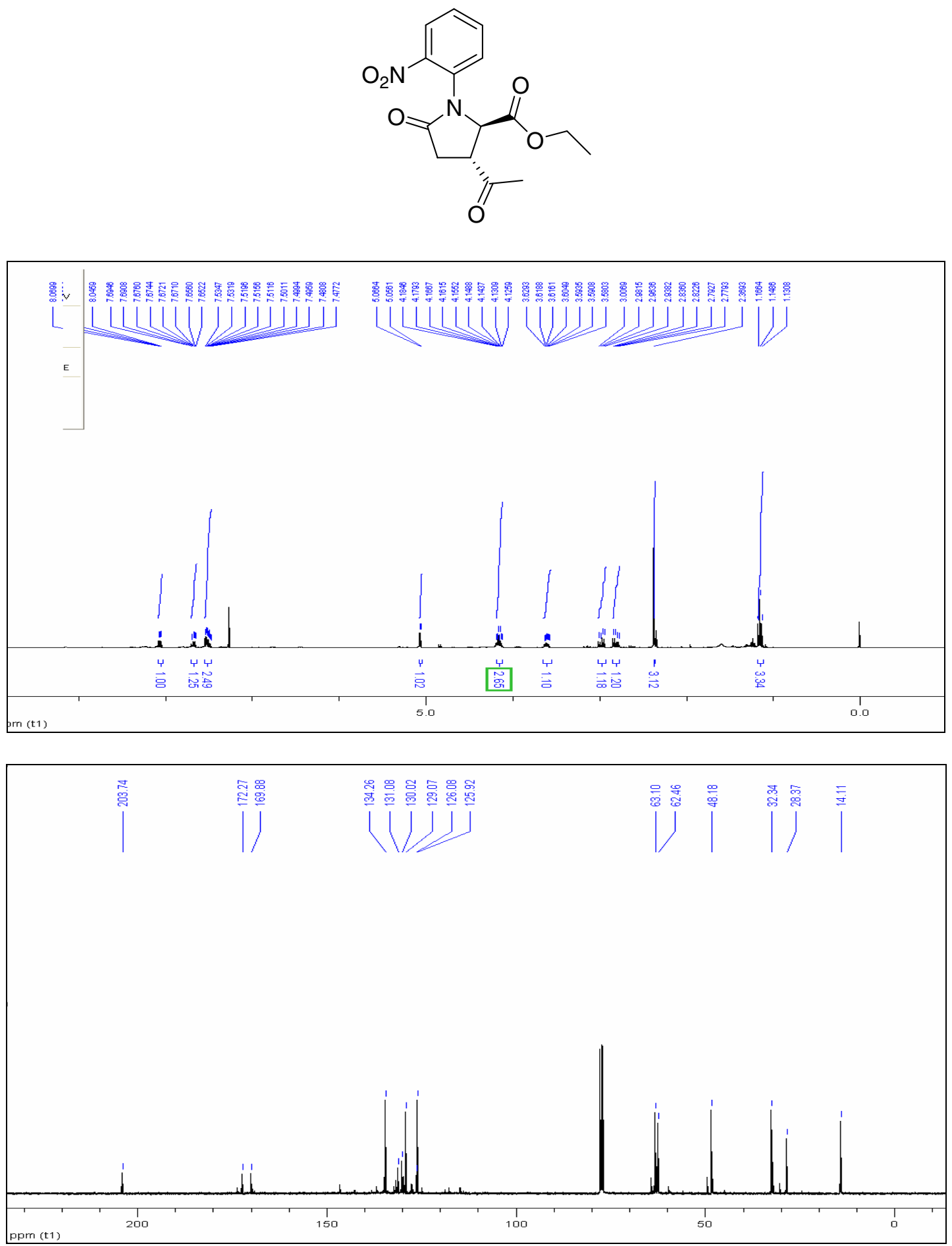
Ethyl 6-methylquinoline 2-carboxylate (5a)
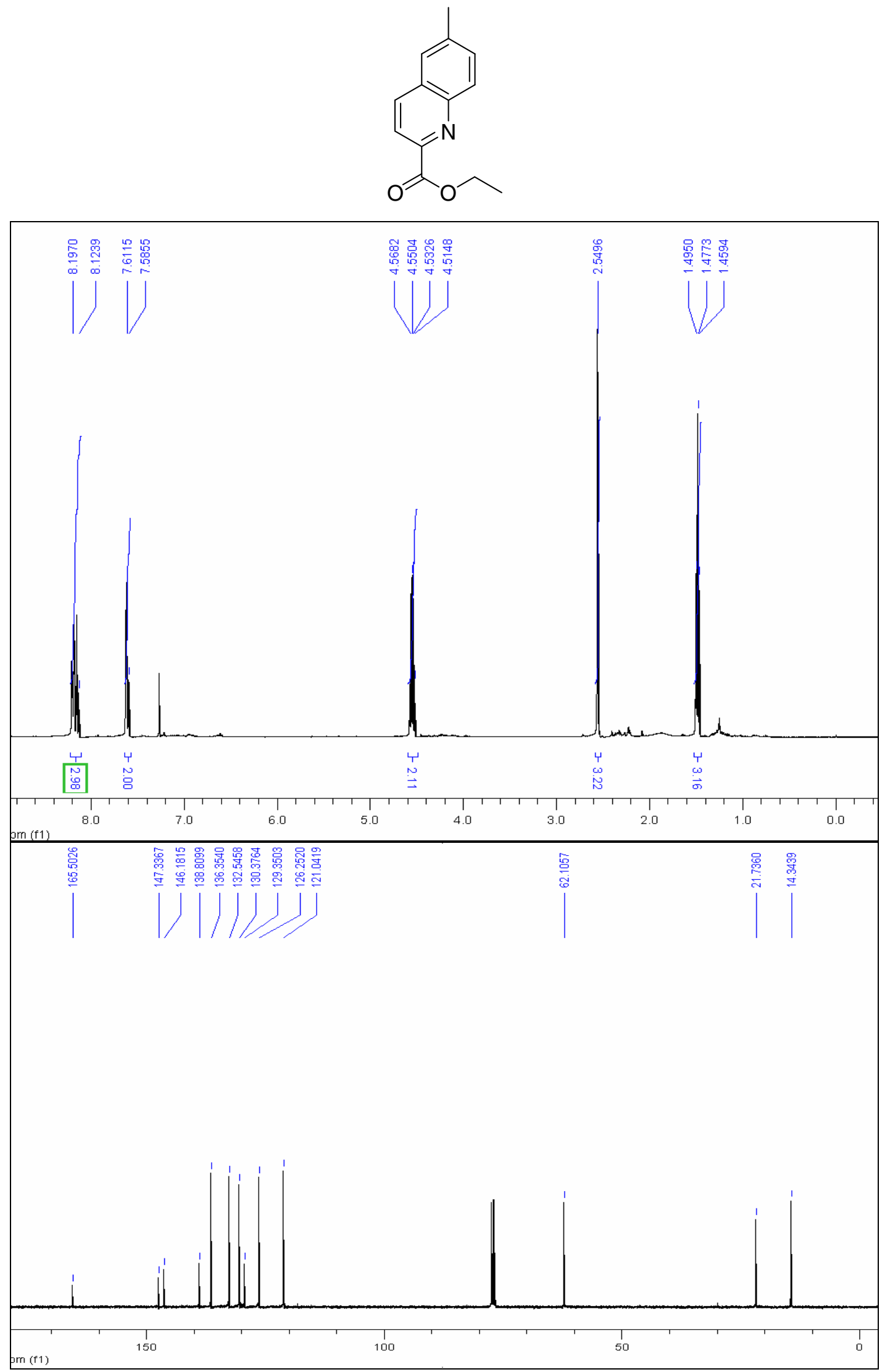
Ethyl 6-methoxyquinoline-2-carboxylate (5b)
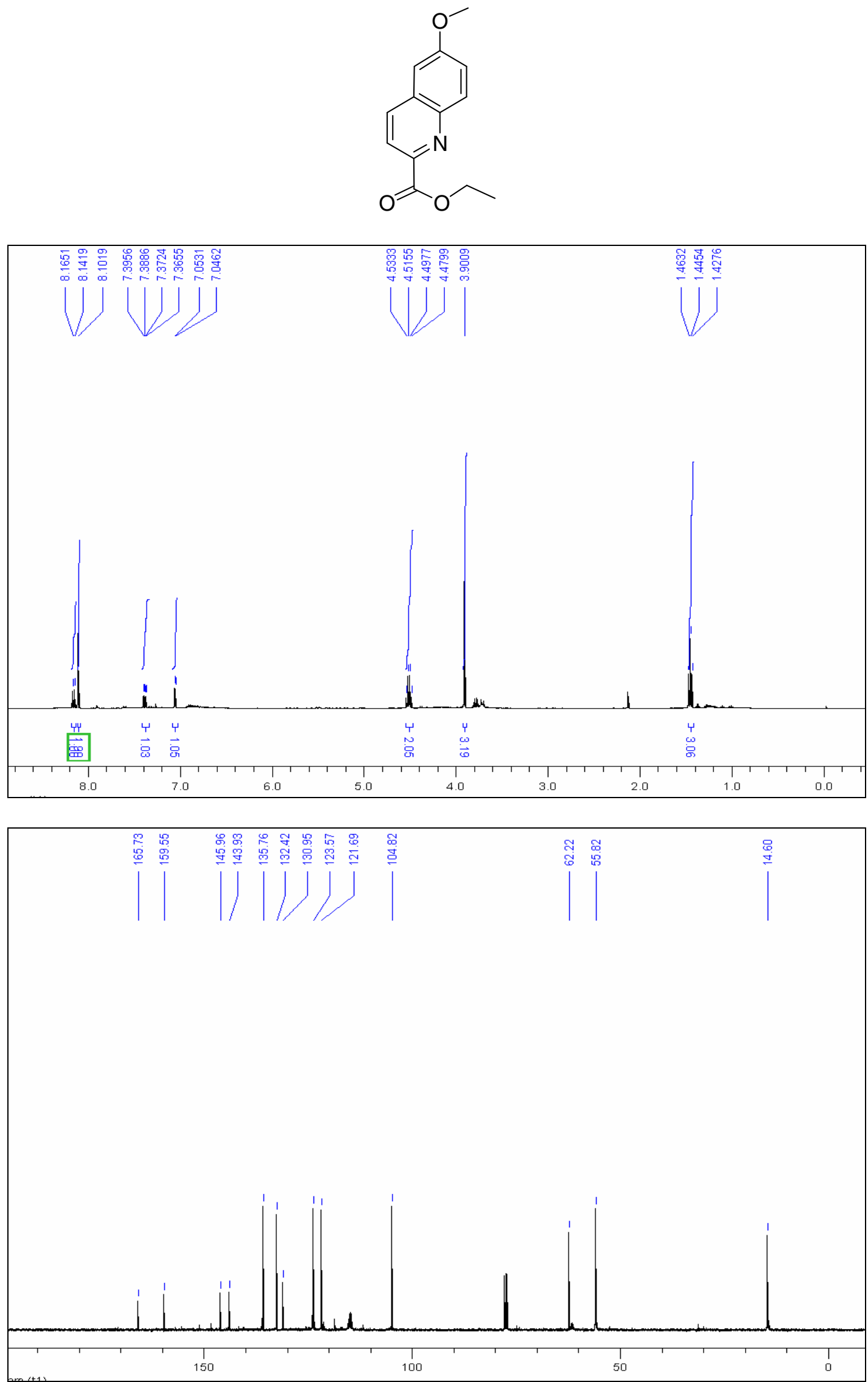
Ethyl 4-oxo-2-( $N$-p-tolylacetamido)pentanoate (6a)

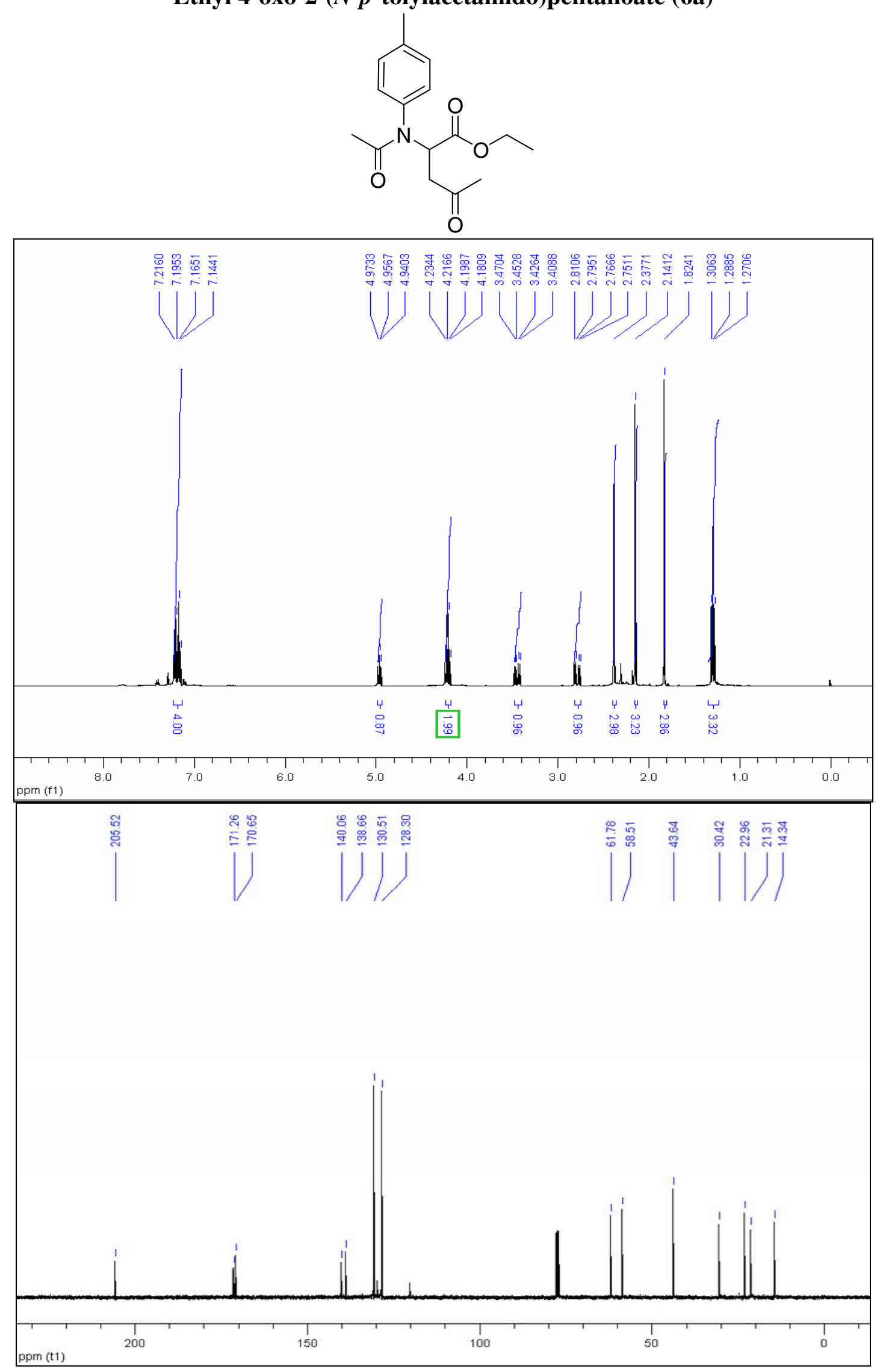


Ethyl 4-[N-(1-ethoxy-1,4-dioxopentan-2-yl)acetamido]benzoate (6b)<smiles>CCOC(=O)c1ccc(N(C(C)=O)C(CC(C)=O)C(=O)OCC)cc1</smiles>
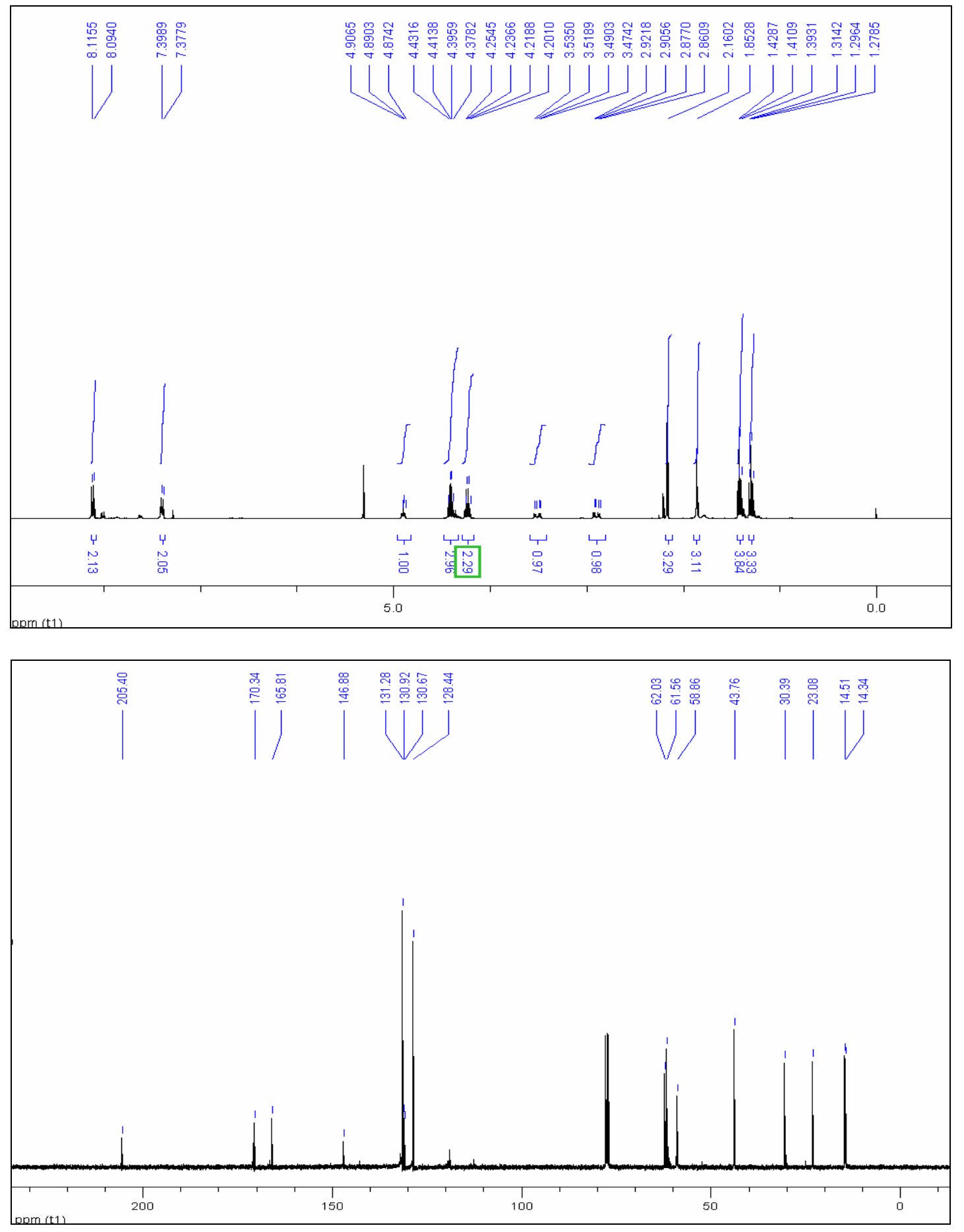

25 
Ethyl 4-(1-ethoxy-1,4-dioxopentan-2-ylamino)benzoate (7b)
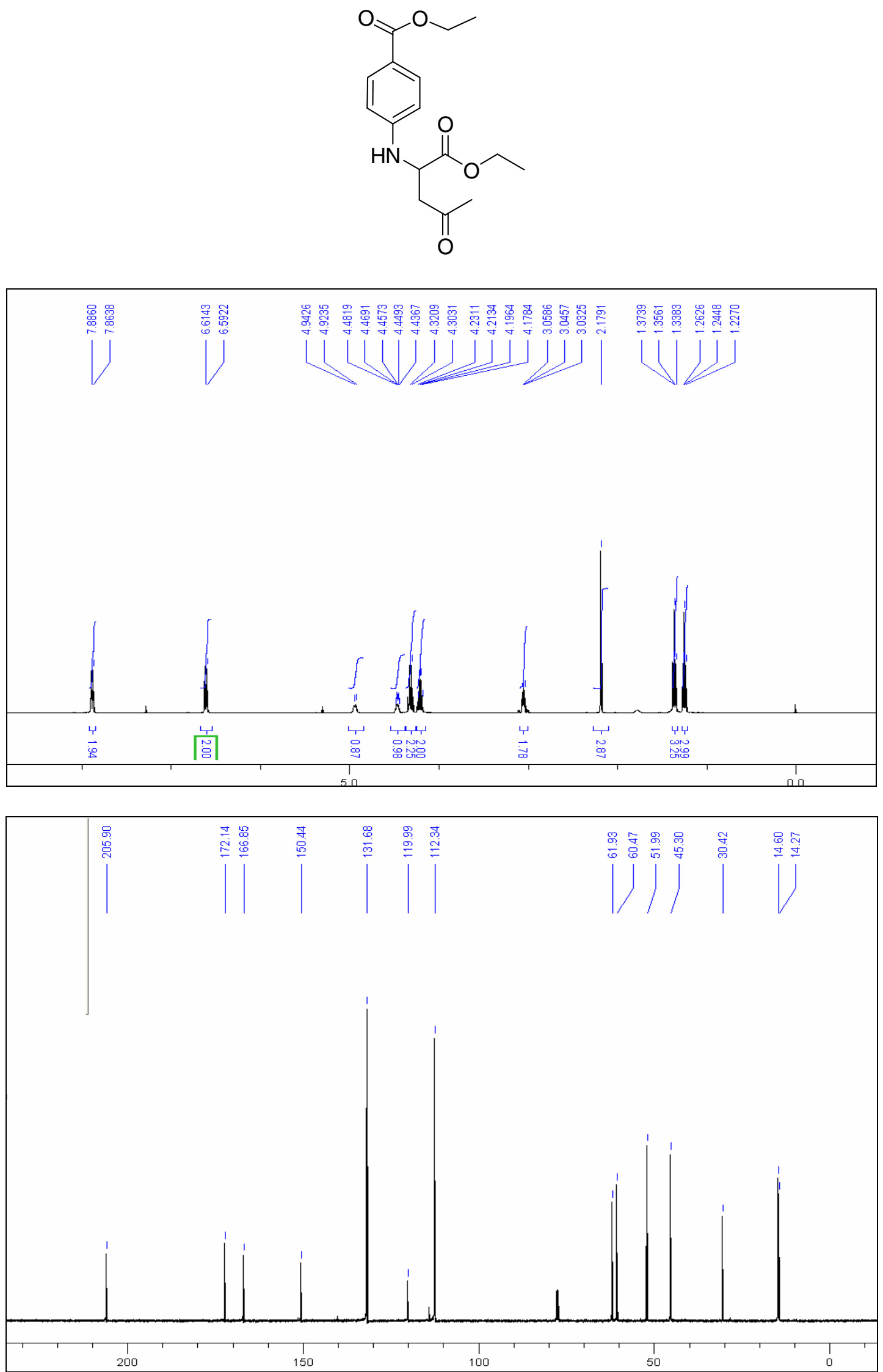
Ethyl 2-(4-nitrophenylamino)-4-oxopentanoate (7c)
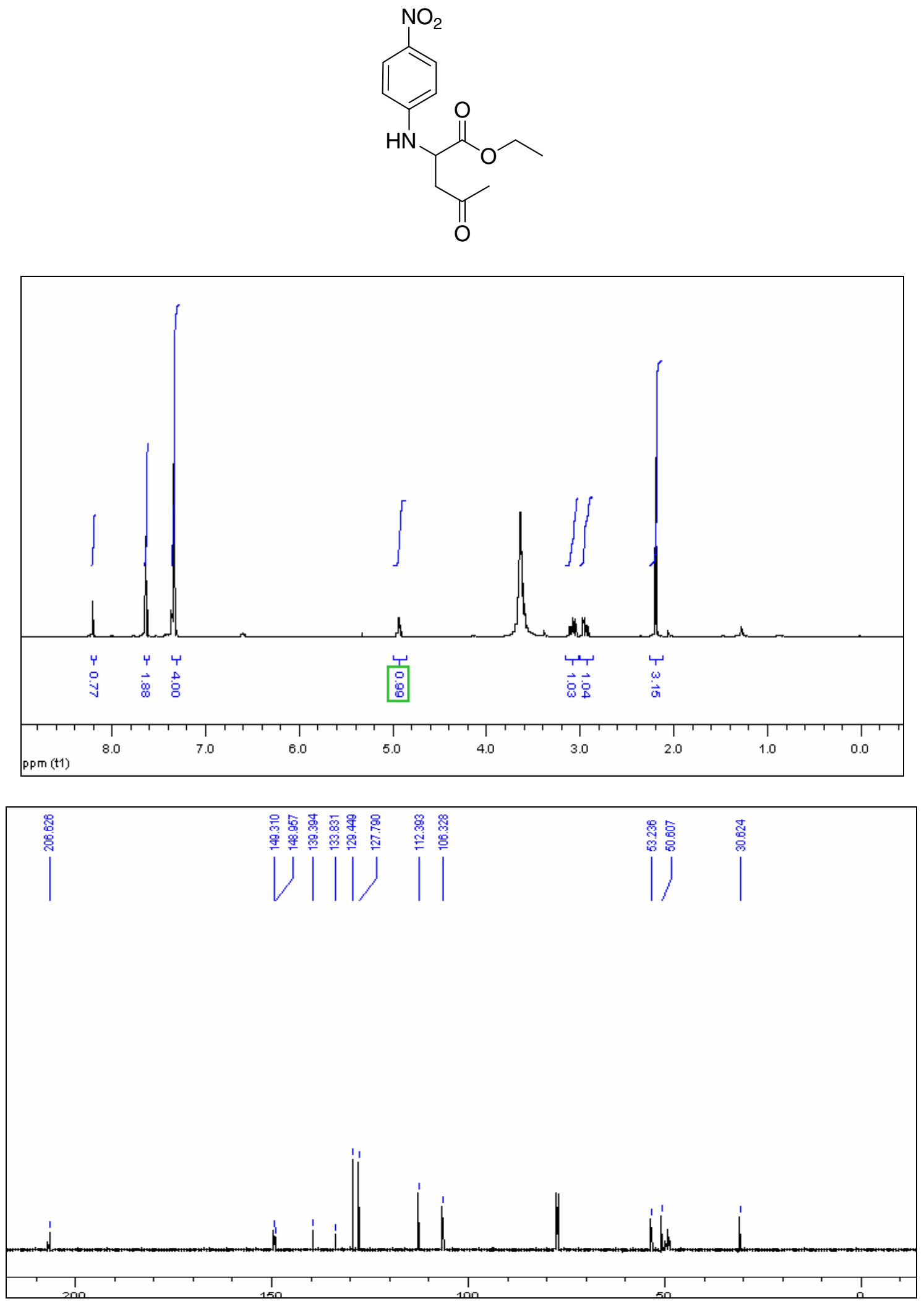
4-(4-Nitrophenyl)-4-(4-nitrophenylamino)butan-2-one (7d)<smiles>CC(=O)CC(Nc1ccc([N+](=O)[O-])cc1)c1ccc([N+](=O)[O-])cc1</smiles>
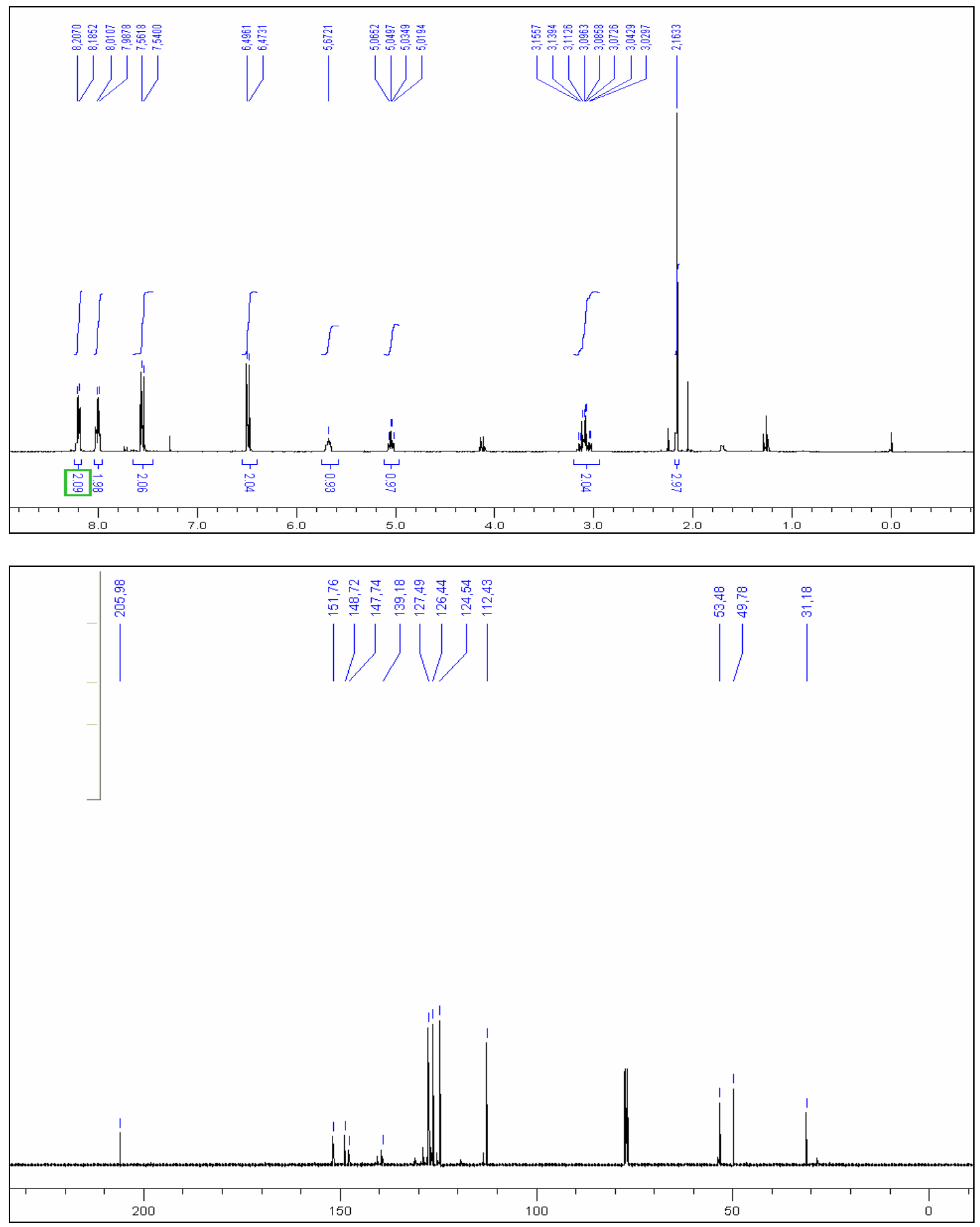
4-(4-Chlorophenyl)-4-(4-nitrophenylamino)butan-2-one (7e)<smiles>CC(=O)CC(Nc1ccc([N+](=O)[O-])cc1)c1ccc(Cl)cc1</smiles>
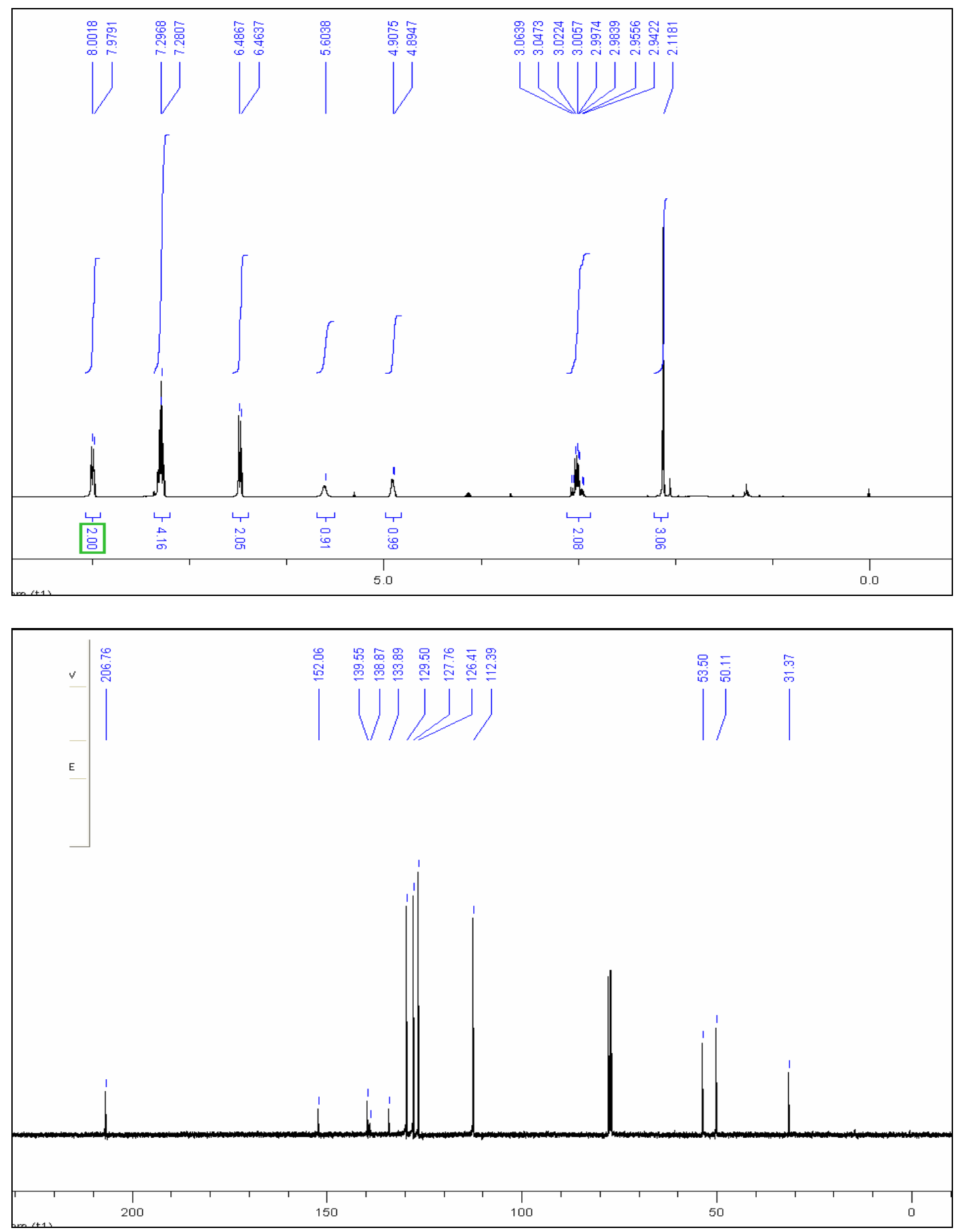

29 
4-(4-Chlorophenyl)-4-(3,5-dinitrophenylamino)butan-2-one (7f)<smiles>CC(=O)CC(Nc1cc([N+](=O)[O-])cc([N+](=O)[O-])c1)c1ccc(Cl)cc1</smiles>

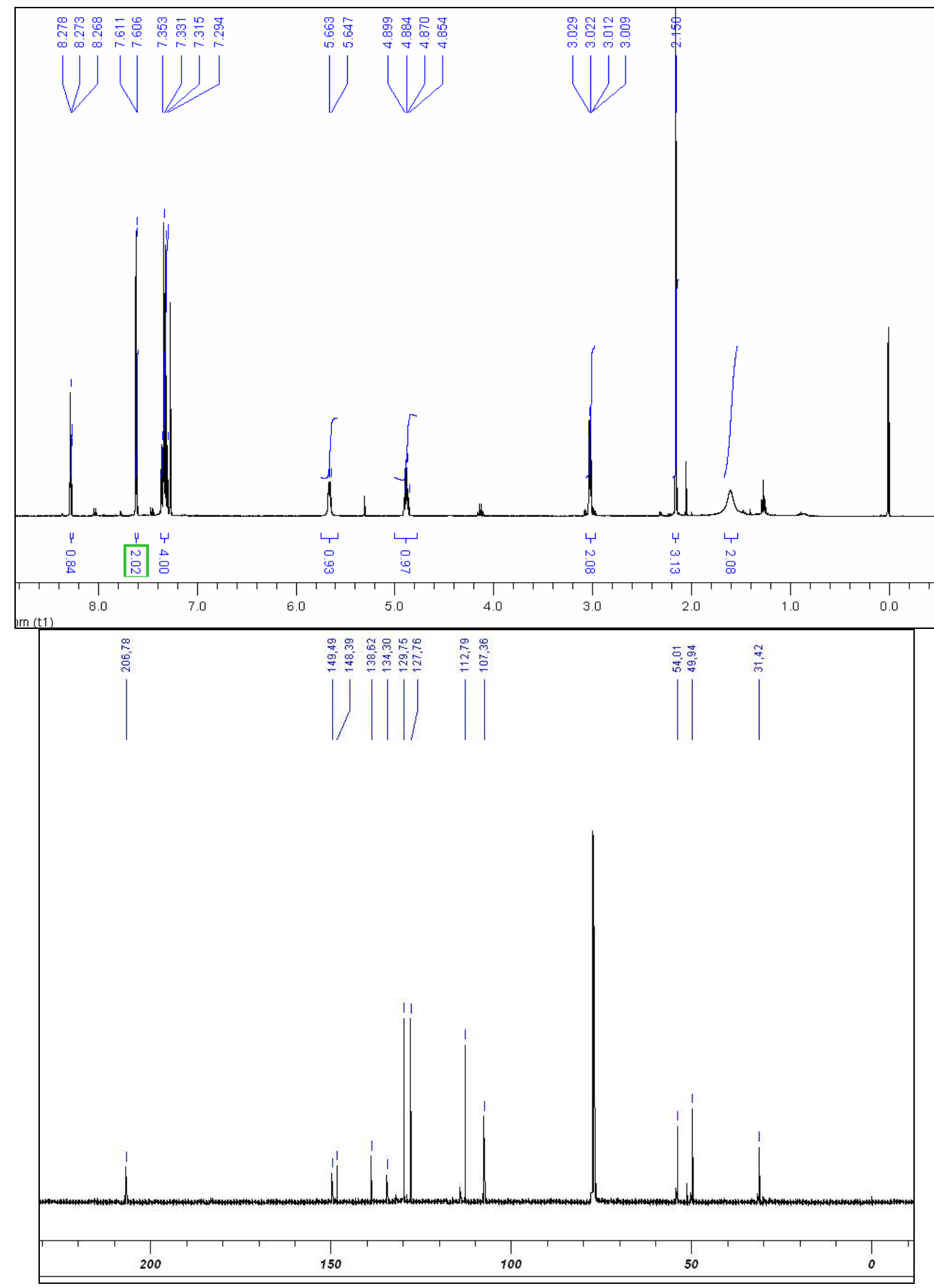

